\title{
A systems analysis of NADH dehydrogenase mutants reveals flexibility and limits of Pseudomonas taiwanensis VLB120's metabolism
}

Nies, Salome C; Dinger, Robert; Chen, Yan; Wordofa, Gossa Garedew; Kristensen, Mette; Schneider, Konstantin; Büchs, Jochen; Petzold, Christopher J.; Keasling, Jay D.; Blank, Lars M

Total number of authors:

11

Published in:

Applied and Environmental Microbiology

Link to article, DOI:

10.1128/aem.03038-19

Publication date:

2020

Document Version

Publisher's PDF, also known as Version of record

Link back to DTU Orbit

Citation $(A P A)$ :

Nies, S. C., Dinger, R., Chen, Y., Wordofa, G. G., Kristensen, M., Schneider, K., Büchs, J., Petzold, C. J., Keasling, J. D., Blank, L. M., \& Ebert, B. E. (2020). A systems analysis of NADH dehydrogenase mutants reveals flexibility and limits of Pseudomonas taiwanensis VLB120's metabolism. Applied and Environmental Microbiology, 86(11), [e03038-19]. https://doi.org/10.1128/aem.03038-19

\section{General rights}

Copyright and moral rights for the publications made accessible in the public portal are retained by the authors and/or other copyright owners and it is a condition of accessing publications that users recognise and abide by the legal requirements associated with these rights.

- Users may download and print one copy of any publication from the public portal for the purpose of private study or research.

- You may not further distribute the material or use it for any profit-making activity or commercial gain

- You may freely distribute the URL identifying the publication in the public portal 


\title{
Systems Analysis of NADH Dehydrogenase Mutants Reveals Flexibility and Limits of Pseudomonas taiwanensis VLB120's Metabolism
}

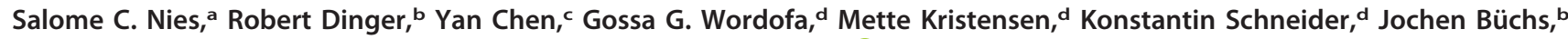 \\ Christopher J. Petzold,c,e Jay D. Keasling, ${ }^{, c, d, e, f, g, h, i, j}$ Lars M. Blank, a (D) Birgitta E. Ebert ${ }^{a, k, l}$
}

\author{
aiAMB-Institute of Applied Microbiology, ABBt-Aachen Biology and Biotechnology, RWTH Aachen University, Aachen, Germany

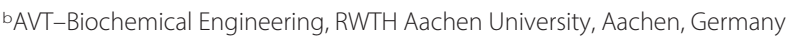 \\ cJoint BioEnergy Institute, Emeryville, California, USA \\ dNovo Nordisk Foundation Center for Biosustainability, Technical University of Denmark, Lyngby, Denmark \\ eLawrence Berkeley National Laboratory, Biological Systems and Engineering Division, Berkeley, California, USA \\ Virtual Institute of Microbial Stress and Survival, Lawrence Berkeley National Laboratory, Berkeley, California, USA \\ gPhysical Biosciences Division, Lawrence Berkeley National Laboratory, Berkeley, California, USA \\ hDepartment of Bioengineering, University of California, Berkeley, California, USA \\ iDepartment of Chemical Engineering, University of California, Berkeley, California, USA \\ Synthetic Biochemistry Center, Institute for Synthetic Biology, Shenzhen Institutes for Advanced Technologies, Shenzhen, Guangdong, China \\ kAustralian Institute for Bioengineering and Nanotechnology (AIBN), University of Queensland, Brisbane, Australia \\ 'CSIRO Future Science Platform in Synthetic Biology, Commonwealth Scientific and Industrial Research Organisation (CSIRO), Canberra, Australia
}

ABSTRACT Obligate aerobic organisms rely on a functional electron transport chain for energy conservation and NADH oxidation. Because of this essential requirement, the genes of this pathway are likely constitutively and highly expressed to avoid a cofactor imbalance and energy shortage under fluctuating environmental conditions. We here investigated the essentiality of the three NADH dehydrogenases of the respiratory chain of the obligate aerobe Pseudomonas taiwanensis VLB120 and the impact of the knockouts of corresponding genes on its physiology and metabolism. While a mutant lacking all three NADH dehydrogenases seemed to be nonviable, the single or double knockout mutant strains displayed no, or only a weak, phenotype. Only the mutant deficient in both type 2 dehydrogenases showed a clear phenotype with biphasic growth behavior and a strongly reduced growth rate in the second phase. In-depth analyses of the metabolism of the generated mutants, including quantitative physiological experiments, transcript analysis, proteomics, and enzyme activity assays revealed distinct responses to type 2 and type 1 dehydrogenase deletions. An overall high metabolic flexibility enables $P$. taiwanensis to cope with the introduced genetic perturbations and maintain stable phenotypes, likely by rerouting of metabolic fluxes. This metabolic adaptability has implications for biotechnological applications. While the phenotypic robustness is favorable in largescale applications with inhomogeneous conditions, the possible versatile redirecting of carbon fluxes upon genetic interventions can thwart metabolic engineering efforts.

IMPORTANCE While Pseudomonas has the capability for high metabolic activity and the provision of reduced redox cofactors important for biocatalytic applications, exploitation of this characteristic might be hindered by high, constitutive activity of and, consequently, competition with the NADH dehydrogenases of the respiratory chain. The in-depth analysis of $\mathrm{NADH}$ dehydrogenase mutants of Pseudomonas taiwanensis VLB120 presented here provides insight into the phenotypic and metabolic response of this strain to these redox metabolism perturbations. This high degree of
Citation Nies SC, Dinger R, Chen Y, Wordofa GG, Kristensen M, Schneider K, Büchs J, Petzold CJ, Keasling JD, Blank LM, Ebert BE. 2020. Systems analysis of $\mathrm{NADH}$ dehydrogenase mutants reveals flexibility and limits of Pseudomonas taiwanensis VLB120's metabolism. Appl Environ Microbiol 86:e03038-19. https://doi.org/10.1128/AEM .03038-19.

Editor M. Julia Pettinari, University of Buenos Aires

Copyright $\odot 2020$ American Society for Microbiology. All Rights Reserved. Address correspondence to Lars M. Blank lars.blanlk@rwth-aachen.de.

Received 6 January 2020

Accepted 18 March 2020

Accepted manuscript posted online 3 April 2020

Published 19 May 2020 
metabolic flexibility needs to be taken into account for rational engineering of this promising biotechnological workhorse toward a host with a controlled and efficient supply of redox cofactors for product synthesis.

KEYWORDS Pseudomonas, NADH dehydrogenase, respiratory activity, oxidative stress, electron transport chain, pseudomonads, redox metabolism

M any industrially relevant molecules, e.g., ethanol, butanediol, or isoprene, are more reduced than the industrially used sugars glucose and sucrose, or alternative, upcoming carbon sources such as xylose or glycerol (1-3). The microbial production of those favored compounds hence is inherently redox limited, i.e., by the supply of reduced redox cofactors, generally NADH or NADPH. This bottleneck has been overcome in some cases, e.g., 1,4-butanediol and 1,3-propanediol production in Escherichia coli $(4,5)$ or L-lysine synthesis in Corynebacterium glutamicum (6). The strategies applied optimized the host metabolism by metabolic engineering $(4,7,8)$ or adapted the process conditions by (co-)feeding reduced substrates (9), applying microaerobic conditions, or using nongrowing cells with reduced competition and cellular demand for the redox cofactor (10-13). Alternatively, microorganisms can be identified that naturally outperform the classic, industrial workhorses with respect to redox cofactor supply. Pseudomonads are outstanding in this regard, as they exhibit a driven-bydemand phenotype which allows strongly enforced metabolic activity under stress conditions with increased energy demand, reported to result in a more than 2-fold carbon uptake rate and an 8 -fold increase of the $\mathrm{NAD}(\mathrm{P}) \mathrm{H}$ regeneration rate relative to standard growth conditions $(12,14,15)$. This behavior holds great promise for using this species for the bioproduction of highly reduced chemicals such as phenol, (S)-styrene oxide, rhamnolipids, and methyl ketones (16-20). Yet, competition is high as the $\mathrm{NAD}^{+} / \mathrm{NADH}$ couple functions as a coenzyme in over 300 oxidation/reduction reactions (21). Pseudomonas strains without apparent fermentative metabolism are obligate aerobes that rely on constitutive activity of the $\mathrm{NADH}$ dehydrogenases to ensure adequate oxidation of $\mathrm{NADH}$ to $\mathrm{NAD}^{+}$. Hence, we argue here that a naturally high NADH oxidation activity might impair the effective fueling of production pathways with reducing equivalents. We here set out to provide an in-depth analysis of the redox metabolism of Pseudomonas taiwanensis VLB120, a strictly aerobic bacterium, focusing on the role and essentiality of the individual NADH dehydrogenases for NADH oxidation and energy conservation.

While the mammalian mitochondrial electron transport chain constitutes only NADH dehydrogenase type 1, a multisubunit enzyme referred to as Nuo or complex 1 (22), which couples the electron transfer to proton translocation and hence contributes to ATP generation (23), aerobic bacteria have developed diverse NADH oxidation capabilities linked to the respiratory chain. Besides the Nuo complex, most species possess one to two isozymes of the non-proton-translocating type 2 dehydrogenase ( $\mathrm{Ndh}$ ), also termed alternative $\mathrm{NADH}$ dehydrogenase, which transfers electrons from NADH to ubiquinone but does not contribute to the membrane potential $(23,24)$. In some species, a third sodium-pumping type 3 dehydrogenase (Nqr) can be found. As with the facultative aerobic yeast Saccharomyces cerevisiae, several bacteria lack the Nuo complex and possess only type 2 dehydrogenases or are reported to mainly rely on the activity of this enzyme for $\mathrm{NADH}$ reoxidation $(25,26)$. Likewise, the genome of $P$. taiwanensis VLB120 encodes two types of NADH dehydrogenases, type 1 (EC 7.1.1.2) and two isoforms of type 2 (EC 1.6.99.3). Type 1 is encoded by the genes PVLB_15600 to PVLB_15660, designated the nuo operon. The two type 2 NADH dehydrogenases are encoded by PVLB_13270 and PVLB_21880, designated ndh-1 and ndh-2, respectively. Ndh-1 and Ndh-2 both consist of a single polypeptide chain.

In the present study, NADH dehydrogenase mutants of $P$. taiwanensis VLB120 were generated and characterized regarding growth, respiratory activity, and transcriptional and proteomic changes to elucidate the impact of redox metabolism perturbation on the cellular physiology. 


\section{RESULTS}

NADH dehydrogenase activity is vital for $P$. taiwanensis but single enzymes of the redundant oxidation system are dispensable. The NADH dehydrogenase type 1 operon encoded by nuoA to nuoN (PVLB_15600 to PVLB_15660) and the two type 2 NADH dehydrogenases encoded by ndh-1 (PVLB_13270) and ndh-2 (PVLB_21880) were successfully deleted from the $P$. taiwanensis VLB120 genome using the I-Scel-based pEMG plasmid (27). The double knockout $\Delta$ ndh-1 $\Delta$ ndh-2 $(\Delta \Delta n d h)$ mutants and $\Delta n u o$ $\Delta$ ndh-1 were successfully obtained, however, several attempts failed to generate the double knockout of $\Delta$ nuo and $\Delta n d h-2$. All gene deletions were confirmed by Sanger sequencing. The five $\mathrm{NADH}$ dehydrogenase mutants demonstrated that the NADH dehydrogenases Nuo, Ndh-1, and Ndh-2 are not essential individually. While the presence of either Nuo or Ndh-2 is sufficient to sustain the viability of $P$. taiwanensis VLB120, Ndh-1 seems to be unable to compensate for the loss of Nuo and Ndh-2. Similarly, it has been reported that single deletions of NADH dehydrogenases in $P$. aeruginosa PAO1 did not result in a growth defect or decrease in NADH oxidation activity, whereas in the double knockout ( $\Delta$ nuolJ $\Delta$ ndh) and triple knockout ( $\Delta$ nuolJ $\triangle$ ndh $\triangle$ nqrABCDEF) the NADH oxidation activity was abolished (28). In conclusion, Nuo and $\mathrm{Ndh}$ account for the total NADH dehydrogenase activity in this Pseudomonas strain. Note that while $P$. aeruginosa is a facultative anaerobe able to respire on nitrate and ferment pyruvate, $P$. taiwanensis VLB120 does not possess the necessary enzymatic makeup for this.

A total loss of NADH dehydrogenase activity in the obligate aerobic $P$. taiwanensis VLB120 strain seems to be lethal, indicating that the strain relies on the presence of these dehydrogenases for $\mathrm{NADH}$ oxidation and that alternative, native NADHconsuming reactions do not suffice to efficiently reoxidize this vital cofactor under the tested conditions.

The $\Delta \Delta$ ndh mutant exhibits a growth-phase-dependent growth defect. $P$. taiwanensis VLB120 and the five NADH dehydrogenase deletion strains $\Delta n d h-1, \Delta n d h-2$, $\Delta \Delta n d h, \Delta n u o$, and $\Delta$ nuo $\Delta n d h-1$ were characterized for growth, glucose utilization, $\mathrm{CO}_{2}$ formation, and oxygen consumption in batch shake-flask experiments. The single NADH dehydrogenase type 2 mutants, $\Delta n d h-1$ and $\Delta n d h-2$, showed the same growth and sugar co-utilization profile as the wild-type $P$. taiwanensis VLB120 (Fig. 1A to C). The loss of the megaplasmid pSTY during NADH dehydrogenase deletions resulted in a growth advantage for the generated mutants, which was determined to result in a $14 \%$ higher growth rate for $P$. taiwanensis VLB120 $\mathrm{pSTY}^{-}$compared to the $\mathrm{pSTY}^{+}$wild type (29). For a comparison of mutants and wild type, the growth rate of the wild type was corrected accordingly and is referred to as $\mu_{\text {recalc. }}$

While the single gene deletion mutants $\Delta n d h-1$ and $\Delta n d h-2$ showed a wild-type physiology (Fig. 1, Table 1 ), the type 2 double mutant $\Delta \Delta$ ndh reproducibly showed two growth phases (Fig. 1D). After wild type-like growth in the first phase, the growth rate dropped drastically in the second growth phase. Interestingly, the strong decrease in the growth rate $(\sim 86 \%)$ was not correlated with an equal reduction in the carbon uptake, which showed a decrease of only $\sim 38 \%$.

Pseudomonas can catabolize glucose either via the phosphorylative or the oxidative pathway. In the latter, a membrane-bound glucose dehydrogenase (Gcd) oxidizes periplasmic glucose to gluconate coupled with the reduction of pyrroloquinoline quinone (PQQ). The phosphorylative pathway starts in the cytoplasm with the phosphorylation of glucose to glucose-6-phosphate catalyzed by the glucokinase (Glk) $(30,31)$.

The mutant $\Delta \Delta$ ndh showed a significant increase in the specific gluconate yield in the early exponential growth phase. The same behavior was observed in the $\Delta$ nuo and $\Delta$ nuo $\Delta$ ndh-1 mutants (Table 1).

Besides the characterization for growth and glucose consumption, the respiratory behavior of the wild type and NADH dehydrogenase mutants was studied (Fig. 2, Fig. $\mathrm{S} 1$ in the supplemental material). Again, only the $\Delta \Delta$ ndh mutant showed a different phenotype characterized by a stagnating oxygen transfer rate (OTR) after $6 \mathrm{~h}$ (Fig. 2B). 
(A) wild type

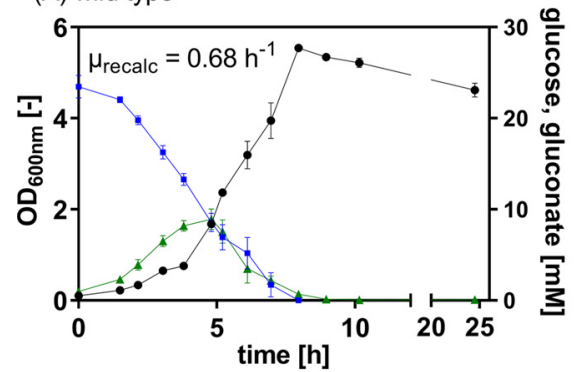

(D) $\Delta \Delta n d h$

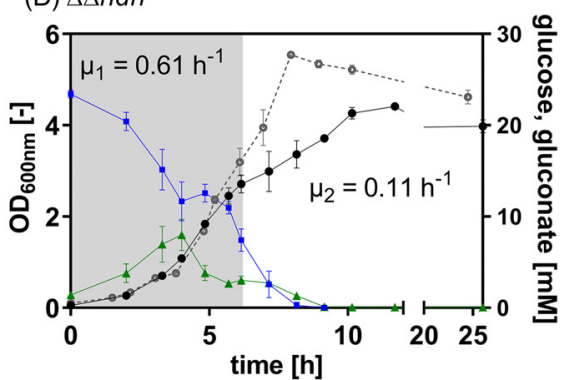

(B) $\Delta n d h-1$

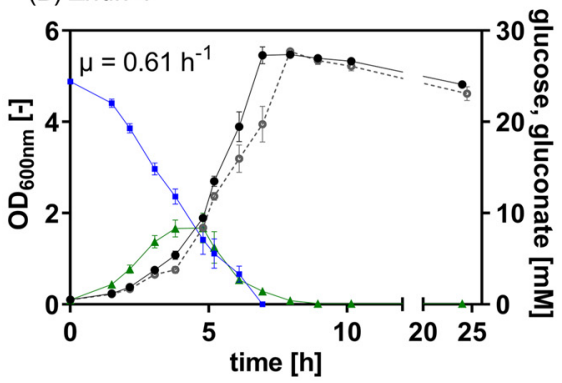

(E) $\Delta n u o$

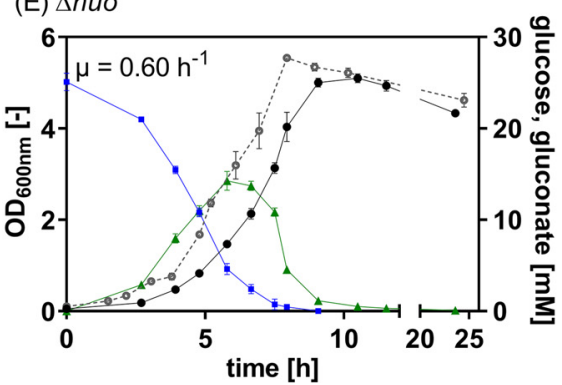

(C) $\Delta n d h-2$

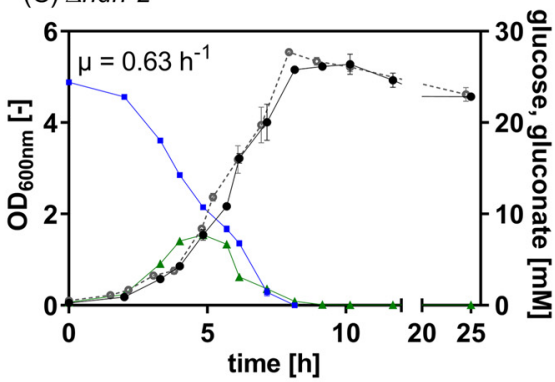

(F) $\Delta$ nuo $\Delta n d h-1$

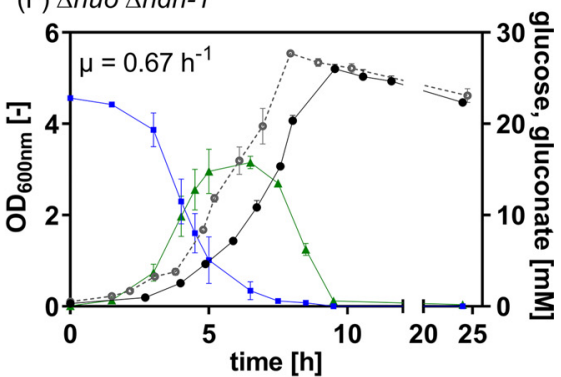

FIG 1 Physiological characterization of $P$. taiwanensis VLB120 wild type (A) and the NADH dehydrogenase deficient mutants $\Delta$ ndh-1 (B), $\Delta$ ndh-2 (C), $\Delta \Delta$ ndh (D), $\Delta$ nuo (E), and $\Delta$ nuo $\Delta$ ndh-1 (F). The strains were cultured in MSM with 25 mM glucose. The OD 600 mm (black circles), glucose levels (blue squares), and gluconate levels (green triangles) were measured over time. The shadowed area in (D) indicates the first growth phase. The data shown are the means of biological

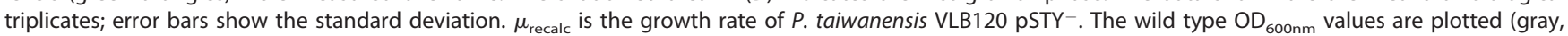
open circles) in graphs (B) to (F) for comparison.

This change in the OTR development is an indication for substrate inhibition, here, potentially by $\mathrm{NADH}$, which cannot be oxidized at the rate required for fast growth. The onset of the reduced specific oxygen uptake rate also correlated well with the change in the growth rate (Fig. 1D).

During growth on glucose, the respiratory quotient (RQ), defined as the ratio of OTR and $\mathrm{CO}_{2}$ transfer rate $(\mathrm{CTR})$, is generally close to one $(32,33)$. Due to the oxidation of glucose to gluconate in the periplasm of Pseudomonas strains, the measured OTR for all tested mutants during the first $6 \mathrm{~h}$ of cultivation was higher than the CTR, resulting in an RQ below 1 (Fig. 2A, Fig. S1). Indeed, the surplus of consumed oxygen, calculated from the sectional integrals between the OTR and CTR ( $\left.\int O T R d t-\int C T R d t\right)$, correlated with the produced gluconate (Table 1, Fig. 2A). During glucose conversion, roughly half

TABLE 1 Calculated carbon uptake, gluconate accumulation, oxygen formation rates, biomass, and the gluconate yield of wild type and $\mathrm{NADH}$ dehydrogenase mutants during exponential growth

\begin{tabular}{|c|c|c|c|c|c|}
\hline Strain & $\begin{array}{l}\text { Mean carbon uptake rate } \\
\left(\mathrm{mmol} \mathrm{g}_{\mathrm{cdw}}{ }^{-1} \mathrm{~h}^{-1}\right) \pm \mathrm{SD}^{a}\end{array}$ & $\begin{array}{l}\text { Mean biomass } \\
\left(\text { g liter }^{-1}\right) \pm \mathrm{SD}^{b}\end{array}$ & $\begin{array}{l}\text { Mean gluconate yield } \\
\left(\mathrm{mmol} \mathrm{g}_{\mathrm{cdw}}{ }^{-1}\right) \pm \mathrm{SD}^{c}\end{array}$ & $\begin{array}{l}\text { Mean gluconate } \\
\text { accumulation } \\
(\mathrm{mM}) \pm \mathrm{SD}^{d}\end{array}$ & $\begin{array}{l}\text { Mean surplus } \mathrm{O}_{2} \\
\text { consumption } \\
(\mathrm{mM}) \pm \mathrm{SD}^{e}\end{array}$ \\
\hline Wild type & $7.3 \pm 0.4$ & $0.7 \pm 0.0$ & $13.7 \pm 2.1$ & $8.9 \pm 1.1$ & $9.2 \pm 1.3$ \\
\hline$\Delta n d h-1$ & $7.9 \pm 0.2$ & $0.7 \pm 0.0$ & $11.6 \pm 1.7$ & $8.6 \pm 1.3$ & $8.1 \pm 0.1$ \\
\hline$\Delta n d h-2$ & $7.2 \pm 0.2$ & $0.6 \pm 0.0$ & $12.8 \pm 1.2$ & $7.7 \pm 0.3$ & $6.3 \pm 0.5$ \\
\hline$\Delta \Delta n d h$ & $8.9 \pm 0.5 / 3.5 \pm 0.2$ & $0.4 \pm 0.0$ & $20.7 \pm 1.1$ & $8.8 \pm 0.4$ & $7.2 \pm 1.6$ \\
\hline$\Delta$ nuo & $7.0 \pm 1.2$ & $0.6 \pm 0.1$ & $25.3 \pm 2.5$ & $14.5 \pm 0.8$ & $12.9 \pm 0.2$ \\
\hline$\Delta$ nuo $\Delta n d h-1$ & $6.1 \pm 0.2$ & $0.8 \pm 0.0$ & $20.9 \pm 1.4$ & $16.1 \pm 1.3$ & $14.1 \pm 3.9$ \\
\hline
\end{tabular}

aFor $\Delta \Delta$ ndh, separate growth rates were determined for phase 1 ( 2 to $6 \mathrm{~h}$ ) and phase 2 ( 6 to $8 \mathrm{~h}$ ). For all other mutants, growth rates were calculated for the exponential phase (between 3 to $4 \mathrm{~h}$ to between 6 to $7 \mathrm{~h}$ after inoculation). SD, standard deviation; $\mathrm{g}_{\mathrm{cdw}}$ grams cell dry weight.

${ }^{b}$ The biomass concentration at the time point of the monitored maximum gluconate concentration according to $d$; shown is the mean of duplicate or triplicate experiments and the corresponding standard deviation.

cThe gluconate yield was calculated by dividing the monitored maximum gluconate concentration according to ${ }^{d}$ with the corresponding biomass concentration according to ${ }^{b}$; shown is the mean of duplicate or triplicate experiments and the corresponding standard deviation. $\mathrm{g}_{\mathrm{cdw}}$ grams cell dry weight.

The gluconate accumulation was determined from offline monitored measurements; shown is the mean of duplicate or triplicate experiments and the corresponding standard deviation.

eThe surplus oxygen consumption was calculated from the sectional integrals between the OTR ( $\left.\mathrm{mmol} \mathrm{liter}^{-1} \mathrm{~h}^{-1}\right)$ and CTR (mmol liter $\left.\mathrm{r}^{-1} \mathrm{~h}^{-1}\right)$ between start of the cultivation and the time point of intersection of CTR and OTR (see Fig. 2); shown is the mean of duplicate experiments and the corresponding standard deviation. 
(A)

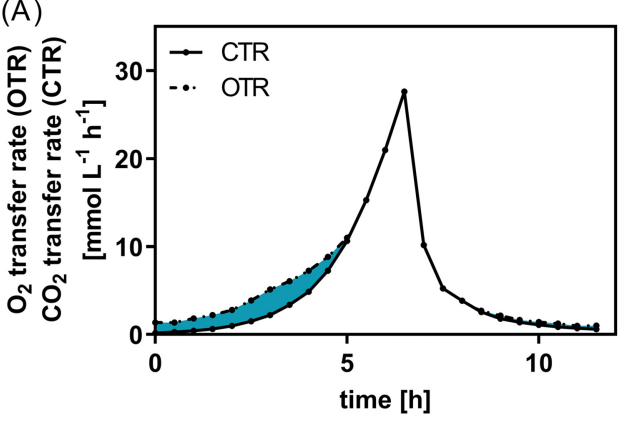

(B)

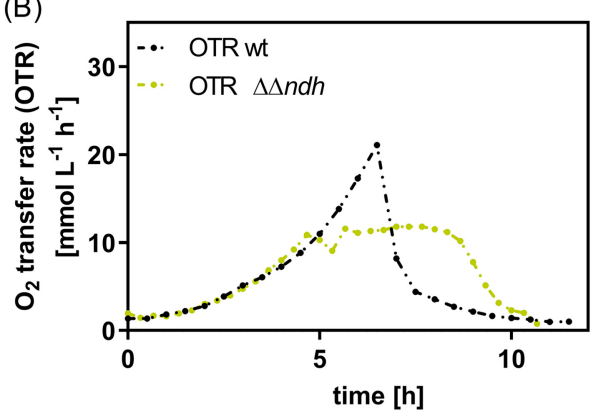

FIG 2 Respiratory activity of $P$. taiwanensis VLB120 and the $\Delta \Delta$ ndh mutant. (A) CTR and OTR rates of the wild-type strain; the highlighted area corresponds to the surplus of consumed oxygen. The area was calculated from the sectional integrals between the OTR (dashed line) and CTR (solid line). (B) Oxygen transfer rates during cultivation of $P$. taiwanensis VLB120 (black dashed line) and mutant $\Delta \Delta$ ndh (green dashed line).

of the overall consumed oxygen was used for the oxidation of glucose to gluconate and the reoxidation of the reduced PQQ formed by the glucose dehydrogenase activity. Consequently, in the glucose phase, the cells can partially uncouple glucose oxidation and energy provision from NADH formation, relieving the dependence on NADH dehydrogenase activity. The $\mathrm{O}_{2}$ and $\mathrm{CO}_{2}$ transfer rates of the $\Delta n d h-2$ and $\Delta \Delta n d h$ mutants (Fig. S1) showed a double peak, which occurred in the same time frame as glucose depletion, and, hence, might be due to the diauxic shift from glucose to gluconate. We assume that the diauxic shift also occurred in the other strains but was not recorded by the measurement frequency of three measurements per hour. The respiratory coefficient on gluconate was close to one for all Pseudomonas strains, indicating that no products other than biomass and $\mathrm{CO}_{2}$ were formed during catabolism of this substrate.

NADH dehydrogenase gene deletions affect expression levels but do not result in altered in vitro NADH oxidation activities. To further elucidate the NADH oxidation activity in the different mutants, and hence, the importance of the three NADH dehydrogenases for oxidizing $\mathrm{NADH}$ and fueling the electron transport chain, we performed in vitro NADH oxidation assays. Inverted membrane vesicles were prepared at early, mid-, and late exponential growth phase, and the NADH oxidation rate was determined from the decrease in absorbance at $340 \mathrm{~nm}$ over time. Note that the SDS-PAGE of the membrane fraction showed up to 21 prominent protein bands (data not shown). Therefore, we cannot exclude the presence of further membrane-bound $\mathrm{NADH}$-dependent enzymes, e.g., the transhydrogenase PntAB, which might have contributed to the measured NADH oxidation rate. However, there is a high probability that the NADH oxidation is very specific for NADH dehydrogenases as most NADHdependent enzymes, e.g., alcohol or aldehyde dehydrogenase, require electron acceptors other than $\mathrm{O}_{2}$. Additional experiments with alternative electron acceptors have not been performed. In the early exponential growth phase, in which none of the strains showed a growth defect, all single mutants possessed NADH oxidation activities at levels similar to the wild type of around $1.2 \mathrm{U} \mathrm{mg}_{\text {protein }}^{-1}$ (Table 2), which is in the range of in vitro rates reported for other organisms (34). Overall, the NADH oxidation rate was rather stable in all mutants, indicating high metabolic flexibility of $P$. taiwanensis VLB120 to maintain redox homeostasis.

To further substantiate this hypothesis, we examined potential changes at the transcriptional level by quantitative PCR (qPCR) on samples taken in the early, mid-, and late exponential growth phase. High-pressure liquid chromatography (HPLC) analysis showed that glucose and/or gluconate were still left when sampling the late exponential growth phase, i.e., the cells were still metabolically active (data not shown). The fold changes were normalized against the wild type in the corresponding growth phase. The single and double deletions of the type $2 \mathrm{NADH}$ dehydrogenase encoding genes (Fig. $3 \mathrm{~A}$ to $\mathrm{C}$ ) had only minor effects (fold changes of $<2$ ) on the remaining NADH 
TABLE 2 Specific NADH oxidation activities of inverted membrane vesicles of $P$. taiwanensis VLB120 wild type and NADH dehydrogenase mutants in the early and late exponential growth phases

\begin{tabular}{lll}
\hline & \multicolumn{2}{l}{$\begin{array}{l}\text { Mean specific NADH oxidation activity } \\
\left(\mathbf{U} \mathrm{mg}_{\text {protein }}{ }^{-1}\right) \pm \mathrm{SD}\end{array}$} \\
\cline { 2 - 3 } Strain & Early exponential & Late exponential \\
\hline Wild type & $1.2 \pm 0.2$ & $0.7 \pm 0.2$ \\
$\Delta$ ndh-1 & $1.3 \pm 0.1$ & $0.5 \pm 0.1$ \\
$\Delta$ ndh-2 & $1.0 \pm 0.2$ & $0.4 \pm 0.1$ \\
$\Delta$ Andh & $1.1 \pm 0.1$ & $0.5 \pm 0.2$ \\
$\Delta$ nuo & $1.2 \pm 0.1$ & $0.5 \pm 0.1$ \\
$\Delta$ nuo $\Delta$ ndh-1 & $0.8 \pm 0.1$ & $0.5 \pm 0.2$ \\
\hline
\end{tabular}

${ }^{a}$ Mean values and standard deviations were determined from independent, biological triplicates.

dehydrogenase gene expression, while the type 1 deletion strains $\Delta$ nuo and $\Delta$ nuo $\Delta$ ndh-1 showed a substantial upregulation of the $n d h-2$ gene expression (Fig. 3D and E). The expression of the $n d h-1$ gene in both $\Delta$ nuo and $\Delta$ nuo $\Delta n d h-1$ was unaffected; we observed only a small increase for mutant $\Delta$ nuo in the early growth phase. This finding suggests that $n d h-2$ is probably the only $\mathrm{NADH}$ dehydrogenase gene that is regulated in response to the cellular $\mathrm{NADH} \mathrm{NAD}^{+}$ratio. The consequent essentiality would further explain why the double deletion of nuo and ndh-2 was lethal. The observation that the $\Delta \Delta n d h$ strain is growth impaired only during mid- to late exponential growth indicates that either the Nuo complex is less active in these phases or that the PQQ-dependent glucose dehydrogenase activity during the early growth phase enables sufficient ATP synthesis independent of NADH dehydrogenase activity.

Double deletion of the type 2 NADH dehydrogenases affects intracellular redox cofactor levels. We found that the NADH oxidation rate was not (or only

(A) $\Delta n d h-1$

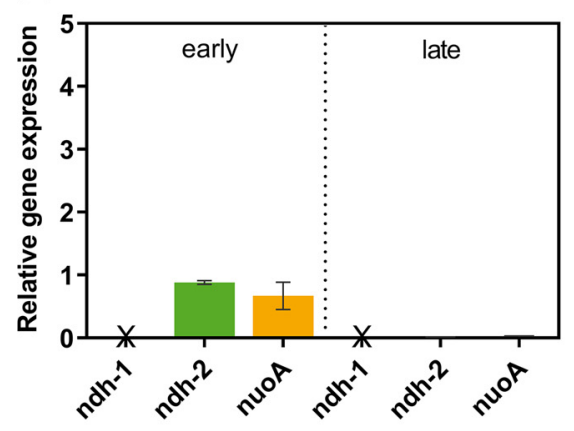

(B) $\Delta n d h-2$

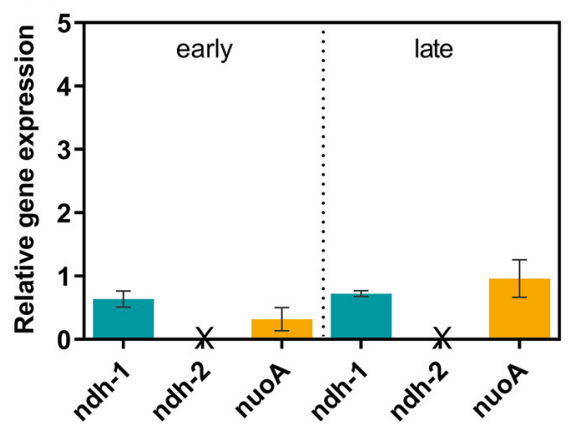

(C) $\Delta \Delta n d h$

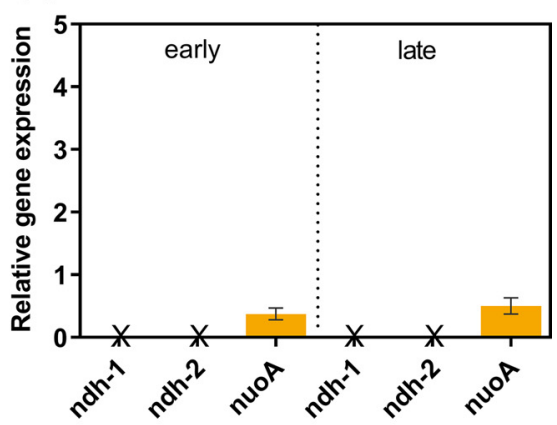

(D) $\Delta$ nuo

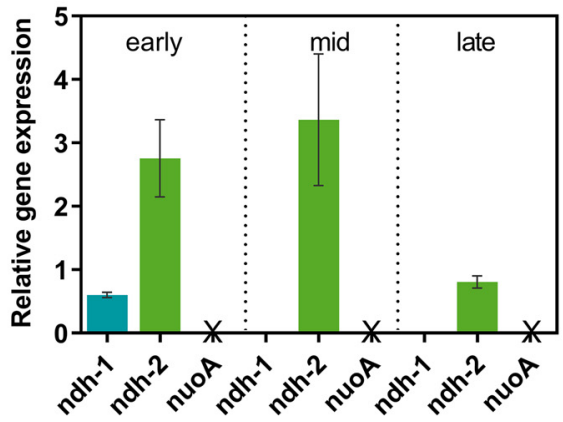

(E) $\Delta$ no $\Delta n d h-1$

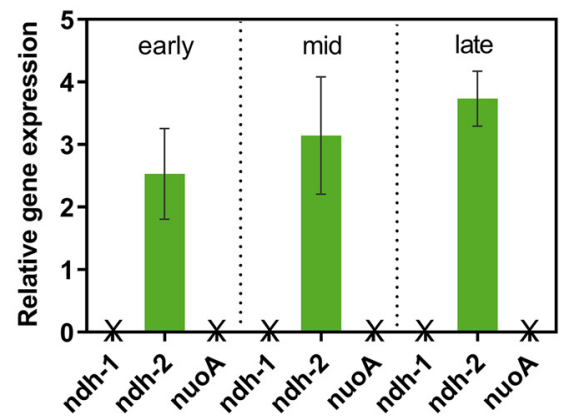

FIG 3 Relative gene expression of the NADH dehydrogenase-encoding genes $n d h-1, n d h-2$, and nuoA in NADH dehydrogenase mutants $\Delta n d h-1$ (A), $\Delta n d h-2$ (B), $\Delta \Delta n d h(C), \Delta n u o(D)$, and $\Delta$ nuo $\Delta n d h-1$ (E) at early, mid-, and late exponential growth phase normalized to the corresponding values of the wild type. mRNA abundance was determined by quantitative PCR. Values were normalized to the relative transcript levels of $P$. taiwanensis VLB120 in the corresponding growth phase. nuoA was used as a proxy for the expression of the nuo operon. Gene deletions in the respective mutants are marked with " $\mathrm{X}$ " and were not analyzed by qPCR. Experiments were performed in biological triplicates. 


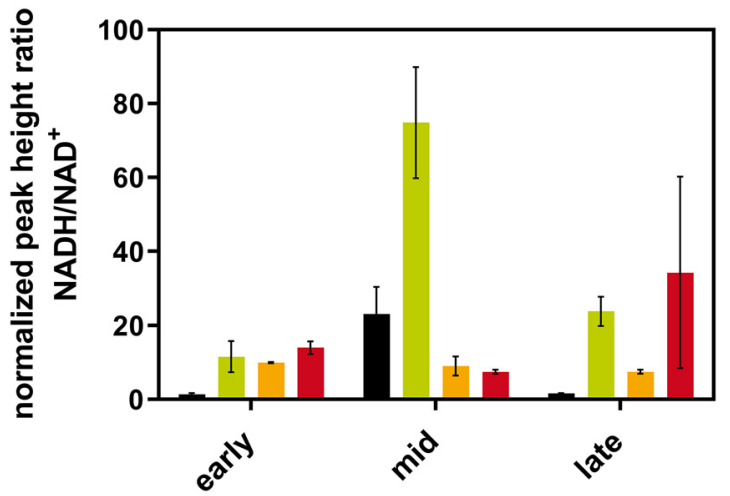

FIG 4 Quantification of the NADH/NAD ${ }^{+}$ratio in the $P$. taiwanensis VLB120 (black) and the NADH dehydrogenase mutants $\Delta \Delta$ ndh (green), $\Delta$ nuo (orange), and $\Delta$ nuo $\Delta$ ndh-1 (red) in early, mid- and late exponential growth phase.

slightly) compromised by the introduced gene deletions, but that the $n d h-2$ level was significantly upregulated, suggesting that its expression is controlled by the redox state of the cell. Moreover, despite that we performed the in vitro enzyme assay with a physiological meaningful NADH concentration of $125 \mu \mathrm{M}$ (35) to mimic in vivo conditions, we cannot exclude differences between these and the actual in vivo NADH dehydrogenase activities of the mutants, as the redox cofactor levels might have been altered.

For this reason, we determined the intracellular abundance of NADH and NAD ${ }^{+}$in the early, mid-, and late exponential growth phase. Since the two single mutants of type 2 dehydrogenases had no growth phenotypes and showed no apparent changes on the transcriptional level, we restricted the analysis to the two double mutants $\Delta \Delta n d h$, and $\Delta$ nuo $\Delta n d h-1$ and the single $\Delta$ nuo deletion mutant.

The $\Delta$ nuo $\Delta n d h-1$ mutant showed a higher NADH/NAD ${ }^{+}$ratio in the late exponential growth phase but also a high variability in the triplicate experiments, thereby curtailing the statistical significance. The double mutant $\Delta \Delta n d h$ had a significantly increased $\mathrm{NADH} / \mathrm{NAD}^{+}$ratio in the mid- and late exponential growth phase compared to the wild type (Fig. 4). This significantly increased $N A D H / N A D^{+}$ratio in $\Delta \Delta$ ndh probably triggered the observed drop in the growth rate in the mid-exponential phase, e.g., because of a potential inhibition of the Nuo complex. To test this hypothesis, we overexpressed the water-forming NADH oxidase (Nox) from Streptococcus pneumoniae (36). Nox is known to be highly specific for $\mathrm{NADH}$, unable to oxidize $\mathrm{NADPH}$, and has been described to produce no toxic hydrogen peroxide (37). The enzyme activity hence results solely in NADH oxidation and is suitable to elucidate the effect of relief from NADH accumulation. The overexpression of nox did not restore the wild-type phenotype but we observed a higher respiratory activity in mutant $\Delta \Delta$ ndh $\mathrm{Nox}^{+}$in comparison to mutant $\Delta \Delta$ ndh (Fig. S3). In contrast to the response of $P$. putida KT2440, elevated Nox activity in the $\Delta \Delta$ ndh mutant did not lead to a decrease in growth rate or biomass yield $(14,38)$.

Proteomic analysis reveals rerouting of the carbon flux in the $\Delta \Delta \boldsymbol{\Delta} \boldsymbol{h} \boldsymbol{h}$ mutant. We further performed shotgun proteomics analysis to explain possible metabolic changes in early, mid-, and late exponential growth phase in P. taiwanensis VLB120 due to NADH dehydrogenase deletions. The relative quantitative data were used to categorize the detected proteins into three groups: (i) significantly upregulated or (ii) downregulated proteins (fold changes of $>2$; adjusted $P$ value $<0.05$ ); and (iii) weak/no effect proteins (fold changes of $<2$ ). The proteins were further grouped into functional categories according to the KEGG database classification (39), e.g., transport, carbohydrate metabolism, or amino acid metabolism (Table S2). The most strongly represented categories are summarized in Fig. 5.

Per the physiological and transcript data, we did not observe significant changes in the proteome for either NADH dehydrogenase type 2 single mutants ( $\Delta$ ndh-1: 9 of 24 
(A) Early

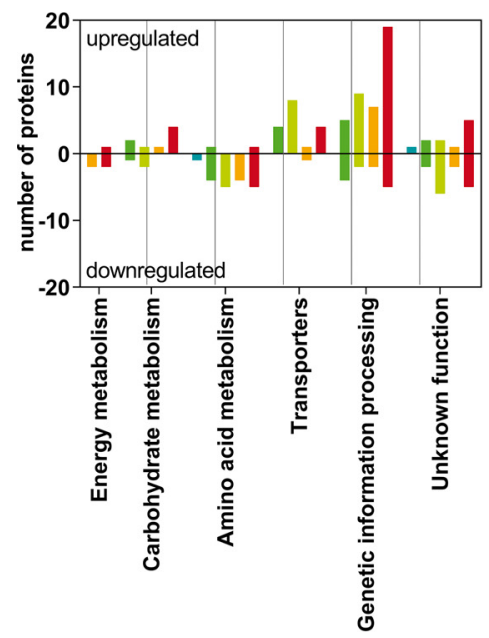

(B) Mid

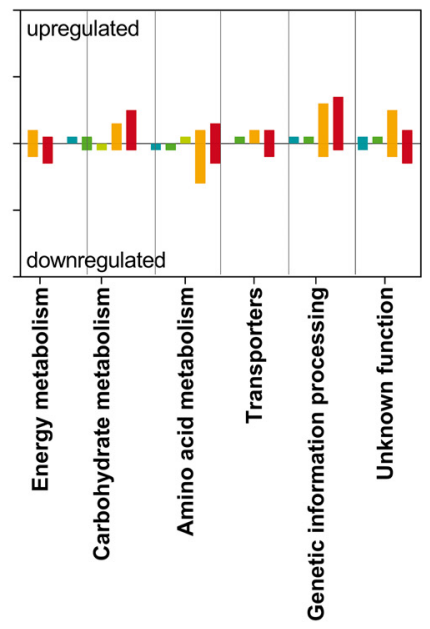

(C) Late

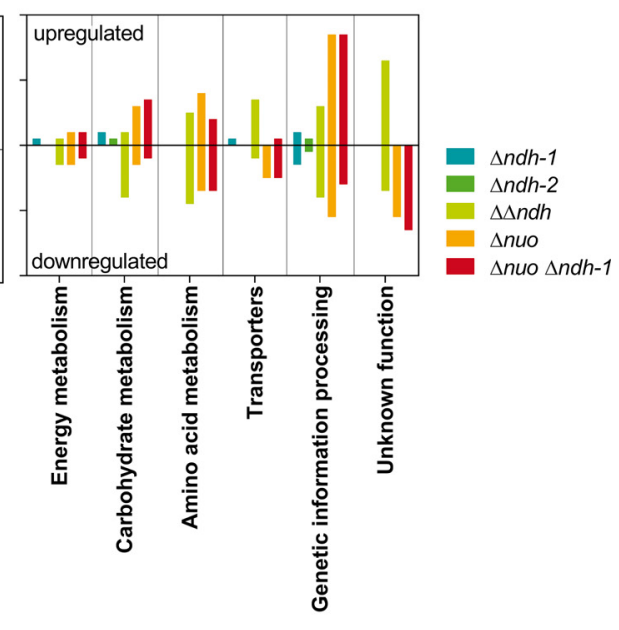

FIG 5 Significant changes at proteome level of $P$. taiwanensis VLB120 NADH dehydrogenase mutants in early (A), mid- (B), and late (C) exponential growth phase relative to the wild type. Proteins are clustered into functional categories according to the KEGG classification system (39). Each bar represents the number of proteins in the depicted category, the abundance of which was either increased or decreased in response to $\mathrm{NADH}$ dehydrogenase deficiency. Experiments were performed in biological triplicates.

proteins significantly up/downregulated; $\Delta$ ndh-2: 8 of 36 proteins significantly up/ downregulated) (Fig. 5, Table S1 and S2). Proteomic changes in both type 1 mutants (Anuo: 50 of 139 proteins significantly up/downregulated; $\Delta$ nuo $\Delta n d h-1: 60$ of 165 proteins significantly up/downregulated) were more significant compared to the type 2 single-gene knockout mutants and very similar to each other (Fig. 5). The double deletion mutant $\Delta \Delta$ ndh showed more alterations in the proteome in the early and late exponential phase (17 and 37 of 107 proteins significantly up/downregulated, respectively) than in the mid-exponential phase ( 2 significantly up/downregulated proteins) (Fig. 5; Table S1).

We next focus on changes observed in the $\Delta \Delta$ ndh mutant for proteins related to carbon uptake, energy generation, and oxidative stress response with respect to highlighting distinct differences from the type $1 \mathrm{NADH}$ dehydrogenase mutants.

The OprB-I porin (PVLB_20075), a carbohydrate selective porin, and the D-gluconate transporter GntT (PVLB_13665) located in the outer and inner membrane, respectively, showed greater increases in the $\Delta \Delta n d h$ mutant during the early and late exponential growth phases, while the glucokinase quantity was strongly reduced in all growth phases (Table 3). These data suggest that the $\Delta \Delta$ ndh mutant strain oxidized glucose via glucose dehydrogenase (Gcd) to gluconate to a greater extent than the wild type. In contrast, the quantity of OprB-I in the type $1 \mathrm{NADH}$ dehydrogenase mutants during the later growth phase was decreased. This change might, however, be explained by the faster glucose depletion in these mutants (Fig. 1).

During the late exponential growth of the $\Delta \Delta$ ndh mutant, all enzymes of the arginine deiminase (ADI) pathway were more strongly expressed, while they were significantly downregulated in the NADH dehydrogenase type 1 mutants (Table 3 ). This pathway catalyzes a three-step conversion of arginine to ornithine, ammonium, and carbon dioxide coupled to ATP generation (40). Likewise, isocitrate lyase (AceA), the first enzyme of the glyoxylate shunt, was upregulated in the $\Delta \Delta$ ndh mutant but downregulated in the $\Delta$ nuo mutant, which instead showed a slight upregulation of the 2-oxoglutarate dehydrogenase complex of the TCA cycle during mid- and late exponential growth. These changes indicate that the mutant $\Delta \Delta$ ndh used the glyoxylate shunt and not exclusively the TCA cycle in the late exponential growth phase.

We further observed remarkable changes in proteins combating oxidative stress. While deletion of the nuo operon ( $\Delta$ nuo and $\Delta$ nuo $\Delta n d h-1)$ resulted in a generally reduced abundance of proteins involved in the oxidative stress response, those mu- 
TABLE 3 Protein abundance in NADH dehydrogenase mutants relative to the wild type

\begin{tabular}{|c|c|c|c|c|c|c|c|c|c|c|}
\hline & & \multicolumn{3}{|c|}{$\Delta \Delta n d h$} & \multicolumn{3}{|c|}{$\Delta$ nuo } & \multicolumn{3}{|c|}{$\Delta n u o \Delta n d h-1$} \\
\hline & & early & mid & late & early & mid & late & early & mid & late \\
\hline $\begin{array}{l}\text { Gene } \\
\text { name }\end{array}$ & $\begin{array}{l}\text { Gene function or } \\
\text { product }^{a}\end{array}$ & \multicolumn{9}{|c|}{ Log2 fold change relative to the wild type ${ }^{b}$} \\
\hline \multicolumn{11}{|c|}{ Carbohydrate metabolism } \\
\hline Pgk & Phosphoglycerate kinase & & & & & & & & & \\
\hline SucC & Succinate-CoA ligase, subunit beta & & & & 0.3 & 0.3 & 0.5 & 0.4 & 0.4 & 0.5 \\
\hline SucD & Succinate-CoA ligase, subunit alpha & & & & & 0.4 & 1.0 & 0.6 & 0.8 & 1.0 \\
\hline SdhB & Succinate dehydrogenase & & & & & & 0.8 & 0.5 & 0.5 & 0.8 \\
\hline Glk & Glucokinase & -3.1 & -2.8 & -2.5 & & & & & & \\
\hline TktA & Transketolase & & & -1.5 & & & & & & \\
\hline AceA & Isocitrate lyase & & & 0.8 & & & -1.3 & & & \\
\hline $\mathrm{Ppc}$ & Phosphoenolpyruvate & & & & \multicolumn{3}{|c|}{1.4} & & & \\
\hline Idh & Isocitrate dehydrogenase & & & & & & 0.6 & & 0.5 & -2.6 \\
\hline Gcd & Quinoprotein glucose & & & & & & 2.2 & & & \\
\hline \multicolumn{11}{|c|}{ Energy metabolism } \\
\hline TsaA & Putative peroxiredoxin & \multirow{4}{*}{\multicolumn{2}{|c|}{-1.0}} & & & \multirow[t]{4}{*}{0.5} & \multirow[t]{4}{*}{0.7} & 0.5 & 0.48 & \multirow[t]{4}{*}{0.79} \\
\hline Fpr-I & Ferredoxin-NADP(+) reductase & & & -1.1 & & & & -3.1 & -1.7 & \\
\hline PP_0235 & Peroxidase & & & 3.2 & & & & & & \\
\hline ChrR & Quinone reductase & & & 2.0 & & & & & & \\
\hline Amino a & cid metabolism & & & & & & & & & \\
\hline ArcA & Arginine deiminase & & & 1.1 & & & -1.9 & & & -1.9 \\
\hline ArcB & Ornithine carbamoyltransferase & & & 1.2 & & & -2.3 & & & -2.3 \\
\hline ArcC & Carbamate kinase & & & 0.8 & -0.6 & & -2.0 & & & -1.5 \\
\hline Transpo & rter/Carbon uptake & & & & & & & & & \\
\hline OprB-I & Porin & 1.4 & & 1.4 & & & -2.5 & & -2.0 & -2.8 \\
\hline GntT & D-gluconate transporter & 0.6 & & 1.2 & & & & 0.9 & & \\
\hline GtsA & Mannose/glucose $A B C$ transporter & 0.8 & & & 0.9 & 0.8 & & 1.0 & 0.7 & \\
\hline GtsD & Mannose/glucose $A B C$ transporter & 0.7 & & & & & & & & \\
\hline Stress $p$ & roteins & & & & & & & & & \\
\hline KatG & Catalase-peroxidase & & & 0.7 & & & -2.3 & & & -2.8 \\
\hline TauA & Taurine ABC transporter & & & 2.4 & & & & & & \\
\hline DnaK & Chaperone protein & & & -0.3 & & & & & & \\
\hline TrxA & Thioredoxin & 0.9 & & 0.6 & & & & & & \\
\hline Colc & or key & & & & & & & & & \\
\hline $\begin{array}{ll}-2 & -1 \\
\end{array}$ & 12 & & & & & & & & & \\
\hline Fold & change & & & & & & & & & \\
\hline
\end{tabular}

${ }^{a}$ Gene function assignment differs from Fig. 5.

${ }^{b}$ Numbers indicate the fold change for upregulated $(+)$ and downregulated $(-)$ proteins relative to the wild type, and the lack of a number indicates no differential production.

cProteins marked with a diamond $(\diamond)$ are discussed in the text.

tants deficient in one of the two type 2 dehydrogenases displayed increased levels of peroxidases and peroxiredoxin proteins. The abundance of the catalase-peroxidase KatG was strongly decreased in both type 1 mutants, whereas it was weakly increased in the $\Delta \Delta$ ndh mutant. Additionally, only the peroxidase encoded by PP_0235 and the quinone reductase ChrR were more highly expressed in the $\Delta \Delta$ ndh mutant, with the latter reported to be induced by superoxide (41), while peroxiredoxin AhpC was weakly upregulated in the single-gene deletion mutants $\Delta n d h-1$ and $\Delta n d h-2$ (Table S2). These 


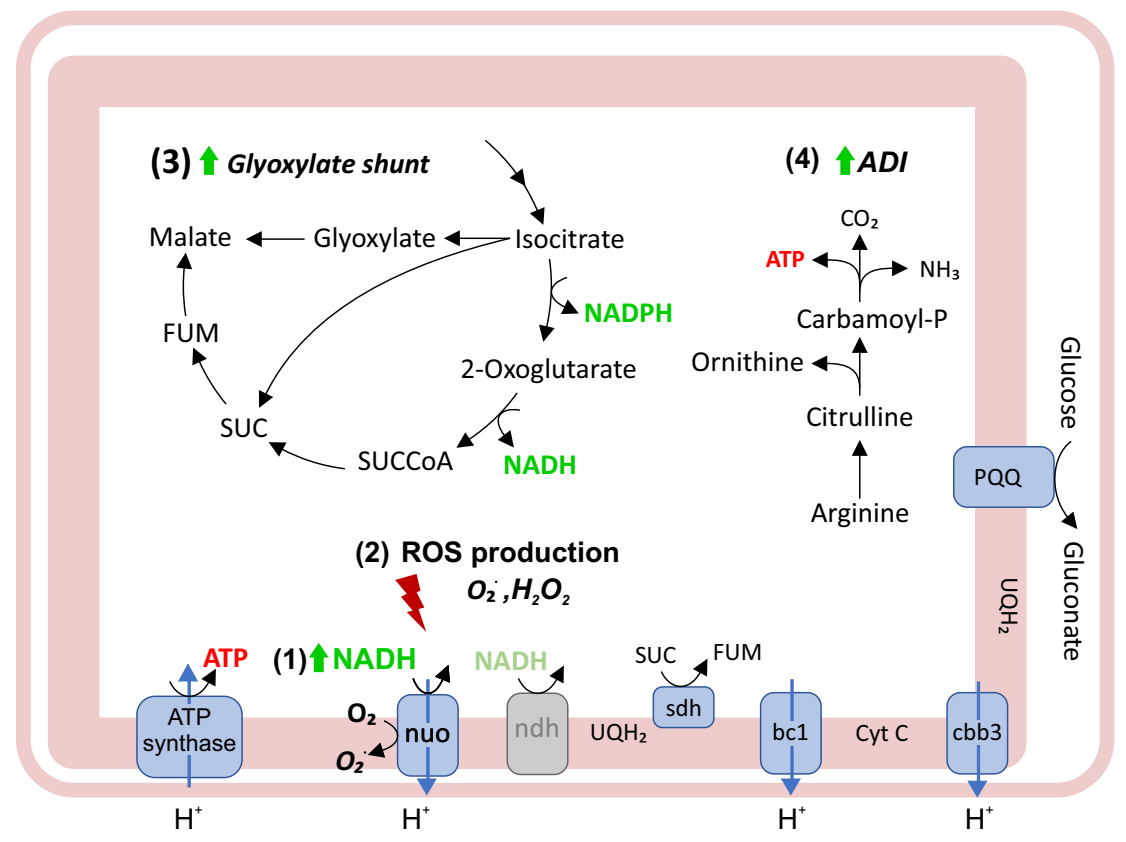

FIG 6 Proposed metabolic changes caused by type 2 NADH dehydrogenase deficiency in $P$. taiwanensis VLB120. An increased NADH/NAD ${ }^{+}$ratio (1) might result in substrate inhibition of the Nuo complex as well as ROS production (2), which is reported for this NADH dehydrogenase $(45,46)$. Rerouting of the flux through the TCA cycle into the glyoxylate shunt (3) reduces redox cofactor formation (48-50) and helps to scavenge reactive oxygen species by glyoxylate $(48,52)$. Limited ATP provision from oxidative phosphorylation can be mitigated by upregulation of the ADI pathway, based on our proteomics data (4) $(58,72)$. The light representation of the $\mathrm{Ndh}$ dehydrogenase indicates deficiency of both isozymes. ETC, electron transport chain; ROS, reactive oxygen species; ADI, arginine deiminase pathway; Nuo, type $1 \mathrm{NADH}$ dehydrogenase; Ndh, type $2 \mathrm{NADH}$ dehydrogenase; Sdh, succinate dehydrogenase; bc1, cytochrome bc 1 (complex III); cbb3, cytochrome $\mathrm{cbb}_{3}$ (complex IV); $\mathrm{QH}_{2}$, ubiquinol; Q, ubiquinone; SUC, succinate, SUCCoA, succinyl-CoA; FUM, fumarate.

findings indicate that the deletion of both type 2 dehydrogenases increases oxidative stress. We determined ROS formation in $P$. taiwanensis VLB120 and mutant $\Delta \Delta$ ndh using the ROS-sensitive dye $2^{\prime}, 7^{\prime}$-dichlorodihydrofluorescein diacetate $\left(\mathrm{H}_{2}-\mathrm{DCFA}\right)$, which is oxidized by ROS to fluorescent DCF. Indeed, we observed an increase in ROS formation in $\Delta \Delta$ ndh concomitant with the reduction in growth (Fig. S2), underlining the proteomic results and strengthening our hypothesis.

\section{DISCUSSION}

This in-depth analysis of NADH dehydrogenase mutants has revealed high metabolic robustness of $P$. taiwanensis VLB120 to a partial loss of the three NADH dehydrogenases, but also the essential nature of residual NADH dehydrogenase activity, as the simultaneous deficiency of Nuo and Ndh-2 was lethal, likely due to inefficient NADH oxidation or ATP provision.

In accordance with the observed phenotypic robustness of most mutants, in vitro $\mathrm{NADH}$ oxidation activities in the mutant strains were not reduced. While this can be explained for those mutants deficient in the nuo operon by the concordant upregulation of ndh-2, no transcriptional changes in NADH dehydrogenase-related genes were observed for the other mutants. In contrast, the mutant with Nuo as the sole NADH dehydrogenase $(\Delta \Delta n d h)$ showed a growth phenotype in the mid-exponential growth phase. While the in vitro NADH oxidation capacity was not altered, several changes in protein and metabolite levels were observed. We have summarized our current model of the potential underlying metabolic changes in Fig. 6. The wild type-like growth of the $\Delta \Delta n d h$ mutant on glucose is likely sustained by periplasmic glucose oxidation to gluconate instead of phosphorylation, which partially uncouples the oxidation of the carbon source from NADH formation. The increased gluconate yield of the $\Delta \Delta n d h$ 
mutant supports this hypothesis. The monitored respiratory activity and the match of gluconate accumulation and surplus oxygen consumption emphasize that no side products other than gluconate were produced to sustain the wild type-like growth. In vitro studies have shown the formation of reactive oxygen species (ROS) such as superoxide $\left(\mathrm{O}_{2}{ }^{-}\right)$and hydrogen peroxide $\left(\mathrm{H}_{2} \mathrm{O}_{2}\right)$ by enzymes of the electron transport chain due to electron leakage to oxygen. Cells activate antioxidant defense systems to combat ROS, which can result in severe cell damage or even death. Nuo (complex I) and

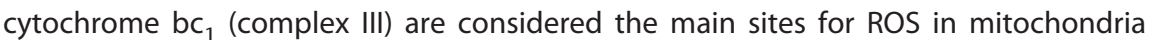
and $P$. fluorescens $(42,43)$ but not in E. coli, which does not possess complex III $(44)$ and rather employs type 2 dehydrogenase. It has further been reported that an oversupply of NADH can enhance ROS production $(45,46)$.

The observed ROS accumulation in $\Delta \Delta$ ndh during mid-exponential growth phase might, hence, be either due to an increased Nuo activity relative to the wild type or to an elevated $\mathrm{NADH} / \mathrm{NAD}^{+}$ratio. In line with this hypothesis, it has been shown that Mycobacterium tuberculosis $\mathrm{NADH}$ dehydrogenase mutants with a similarly elevated $\mathrm{NADH} / \mathrm{NAD}^{+}$ratio were more susceptible to (additional) oxidative stress than those with a lower NADH/NAD ${ }^{+}$ ratio (47). ROS induces stress, but a further explanation for the reduced growth and respiratory activity may lie in the potential NADH inhibition of metabolic enzymes. However, while the latter increased upon nox expression, Nox-mediated NADH oxidation did not restore growth, indicating that further limitations remained.

The activation of the glyoxylate shunt in $\Delta \Delta n d h$, as indicated by the proteome data, might contribute to stress reduction in two ways. First, this shortcut of the TCA cycle bypasses NAD(P)H-producing steps (48-50). For Pseudomonas putida KT2440, the production of a surplus of NADPH during growth on glucose has been reported (51) and it was speculated that the excess NADPH is converted to NADH by the transhydrogenase PntAB, which has been shown to be expressed in this strain under similar growth conditions (38). Assuming PntAB transhydrogenase activity in $P$. taiwanensis, which is equipped with the respective genes, attenuated $\mathrm{NADP}^{+}$reduction would, hence, result in an overall reduced NADH formation. Second, the glyoxylate formed by the isocitrate lyase AceA activity, which was upregulated in $\Delta \Delta$ ndh, can react with hydrogen peroxide to produce formate and $\mathrm{CO}_{2}$ $(48,52)$. This ROS combating strategy has been reported for Pseudomonas aeruginosa, Burkholderia cenocepacia, and Staphylococcus aureus, even though S. aureus has no functional glyoxylate shunt $(48,53-55)$. Note, however, that neither increased formate dehydrogenase abundance nor formate accumulation was observed in the $\Delta \Delta$ ndh mutant.

The deletion of nuo and the accompanying higher Ndh-2 activity did not result in a similar stress response, which is in accordance with corresponding $M$. tuberculosis mutants (47).

A probable energy shortage due to reduced respiratory activity might have been counteracted by ATP generation via the arginine deiminase (ADI) pathway, which seems to be activated in $\Delta \Delta$ ndh according to the proteome data. This pathway generates $1 \mathrm{~mol}$ ATP per mol arginine $(40,56)$ and has been reported to be activated upon energy depletion in lactic bacteria (57) and pseudomonads, e.g., in P. putida DOT-T1E under energy-demanding solvent stress conditions $(58,59)$ and in $P$. aeruginosa under oxygen limiting conditions (40).

In this study, we showed high metabolic flexibility of $P$. taiwanensis VLB120 to interventions in the redox metabolism, which confers robust phenotypic behavior by a possible rerouting of metabolic fluxes. This metabolic adaptability and phenotypic robustness can be advantageous for biocatalysis but simultaneously challenging because it impedes the prediction of mutant behavior and can lever out metabolic engineering efforts. Hence, to effectively turn this promising microbe into a controllable, biotechnological workhorse, further biological and physiological system analyses, such as ${ }^{13} \mathrm{C}$ metabolic flux analysis, are needed.

Strains, media, and culture conditions. Bacterial strains used in this study are listed in Table 4. Strains were propagated in lysogeny broth (LB) containing $10 \mathrm{~g} \mathrm{liter}^{-1}$ peptone, $5 \mathrm{~g} \mathrm{liter}^{-1}$ sodium 
TABLE 4 Bacterial strains and plasmids used in this study

\begin{tabular}{|c|c|c|}
\hline Strain or plasmid & Relevant characteristics ${ }^{a}$ & Reference \\
\hline \multicolumn{3}{|l|}{ E. coli } \\
\hline HB101 pRK2013 & SmR, hsdR-M+, proA2, leuB6, thi-1, recA; bears plasmid pRK2013 & (73) \\
\hline $\mathrm{DH} 5 \alpha \mathrm{pSW}-2$ & $\mathrm{Gm}^{\mathrm{r}}$, DH5 $\alpha$ bearing pSW-2 & $(27)$ \\
\hline DH5 $\alpha$ p pir1 pEMG_ko_ndh2 & $\mathrm{Km}^{\mathrm{r}}$, PVLB_21880 deletion plasmid & This study \\
\hline DH5 $\alpha$ p pir1 pEMG_ko_nuo & Kmr, PVLB_15600-15660 deletion plasmid & This study \\
\hline \multicolumn{3}{|l|}{ Plasmids } \\
\hline pS2311·Nox & $\mathrm{Km}^{\mathrm{r}}$; derivative of vector pSEVA2311 with the nox gene from S. pneumoniae & (36) \\
\hline \multicolumn{3}{|l|}{ P. taiwanensis } \\
\hline VLB120 $\Delta n d h-2$ & $\Delta n d h-2$ (PVLB_21880), pSTY- & This study \\
\hline VLB120 $\Delta \Delta n d h$ & $\Delta \Delta n d h($ PVLB_13270, PVLB_21880), pSTY- & This study \\
\hline VLB120 $\Delta$ nuo & $\Delta n u o($ PVLB_15600-15660), pSTY- & This study \\
\hline VLB120 $\Delta$ nuo $\Delta n d h-1$ & dndh-1 (PVLB_13270), $\Delta$ nuo (PVLB_15600-15660), pSTY- & This study \\
\hline VLB120 pS2311·Nox & $\mathrm{Km}^{\mathrm{r}}$, VLB120 bearing pS2311·Nox & \\
\hline VLB120 $\Delta \Delta n d h \mathrm{pS} 2311 \cdot$ Nox & $\mathrm{Km}^{\mathrm{r}}, \Delta \Delta$ ndh (PVLB_13270, PVLB_21880), pSTY- bearing pS2311·Nox & This study \\
\hline
\end{tabular}

${ }^{a} \mathrm{Gm}^{r}$, gentamicin resistance; $\mathrm{Km}^{r}$, kanamycin resistance.

chloride, and $5 \mathrm{~g} \mathrm{liter}^{-1}$ yeast extract (60). Cetrimide agar (Sigma-Aldrich, St. Louis, MO, USA) was used after mating procedures to select for Pseudomonas. Growth and characterization experiments were performed using mineral salt medium (MSM) (61) containing $3.88 \mathrm{~g} \mathrm{liter}^{-1} \mathrm{~K}_{2} \mathrm{HPO}_{4}, 1.63 \mathrm{~g}$ liter ${ }^{-1}$ $\mathrm{NaH}_{2} \mathrm{PO}_{4}, 2 \mathrm{~g} \mathrm{liter}^{-1}\left(\mathrm{NH}_{4}\right)_{2} \mathrm{SO}_{4}, 0.1 \mathrm{~g} \mathrm{liter}^{-1} \mathrm{MgCl}_{2} \cdot 6 \mathrm{H}_{2} \mathrm{O}, 10 \mathrm{mg} \mathrm{liter}^{-1}$ EDTA, $2 \mathrm{mg}$ liter ${ }^{-1} \mathrm{ZnSO}_{4} \cdot 7 \mathrm{H}_{2} \mathrm{O}$, $1 \mathrm{mg}$ liter ${ }^{-1} \mathrm{CaCl}_{2} \cdot 2 \mathrm{H}_{2} \mathrm{O}, 5 \mathrm{mg} \mathrm{liter}{ }^{-1} \mathrm{FeSO}_{4} \cdot 7 \mathrm{H}_{2} \mathrm{O}, 0.2 \mathrm{mg} \mathrm{liter}{ }^{-1} \mathrm{Na}_{2} \mathrm{MoO}_{4} \cdot 2 \mathrm{H}_{2} \mathrm{O}, 0.2 \mathrm{mg} \mathrm{liter}^{-1} \mathrm{CuSO}_{4} \cdot 5$ $\mathrm{H}_{2} \mathrm{O}, 0.4 \mathrm{mg}$ liter ${ }^{-1} \mathrm{CoCl}_{2} \cdot 6 \mathrm{H}_{2} \mathrm{O}, 1 \mathrm{mg} \mathrm{liter}-1 \mathrm{MnCl}_{2} \cdot 2 \mathrm{H}_{2} \mathrm{O}$ supplemented with $25 \mathrm{mM}$ glucose. For the preparation of solid LB, $1.5 \%$ agar was added to the medium. For plasmid maintenance and in the gene deletion procedure, antibiotics were added to the medium as required. Gentamicin and kanamycin were used at concentrations of $25 \mathrm{mg} \mathrm{liter}^{-1}$ and $50 \mathrm{mg} \mathrm{liter}^{-1}$, respectively. Because of the leaky expression of nox from plasmid pS2311, P. taiwanensis VLB120 strains bearing plasmid pS2311 were grown without the addition of the inducer cyclohexanone.

Batch-flask experiments were performed in $50 \mathrm{ml}$ medium in 500-ml flasks under oxic conditions on a horizontal rotary shaker with a throw of $50 \mathrm{~mm}$ and frequency of $300 \mathrm{rpm}$. E. coli was grown at $37^{\circ} \mathrm{C}$, Pseudomonas at $30^{\circ} \mathrm{C}$. The chemicals used in this work were obtained from Carl Roth (Karlsruhe, Germany), Sigma-Aldrich (St. Louis, MO, USA), or Merck (Darmstadt, Germany) unless stated otherwise. The main cultures were inoculated from liquid precultures to an approximate $\mathrm{OD}_{600 \mathrm{~nm}}$ of 0.05 . All experiments were performed in biological triplicates unless stated otherwise.

Plasmid cloning and generation of deletion strains. Genomic DNA of $P$. taiwanensis VLB120 was isolated using the High Pure PCR template preparation kit (Hoffmann-La-Roche, Basel, Switzerland). Upstream (TS1) and downstream (TS2) regions with a length of 400 to $800 \mathrm{bp}$ flanking the specific target gene were amplified using Q5 high-fidelity polymerase (New England Biolabs, Ipswich, MA, USA). Primers were ordered as unmodified DNA oligonucleotides from Eurofins Genomics (Ebersberg, Germany) and are listed in Table 5. The suicide delivery vector pEMG was isolated using the NEB Monarch plasmid miniprep kit (New England Biolabs, Ipswich, MA, USA). The isolated plasmid was digested with restriction enzymes purchased from New England Biolabs (Ipswich, MA, USA). For plasmid construction, Gibson Assembly using NEB Builder Hifi DNA assembly (New England Biolabs, Ipswich, MA, USA) was used. Plasmids were transformed into electrocompetent $E$. coli $\mathrm{DH} 5 \alpha \lambda$ pir 1 via electroporation (62). Transformants and chromosomally engineered Pseudomonas were screened by colony PCR using OneTaq $2 \times$ Master Mix (New England Biolabs, Ipswich, MA, USA). The cell material was lysed in alkaline polyethylene glycol for enhanced colony PCR efficiency as described previously (63).

Targeted gene deletions were performed using the I-Scel-based system developed by MartinezGarcia and de Lorenzo (27). The conjugational transfer of the mobilizable knockout plasmids from $E$. coli $\mathrm{DH} 5 \alpha \lambda$ pir1 to Pseudomonas was performed via triparental patch mating (16). After conjugation, the pSW-2 plasmid encoding the I-Scel endonuclease was conjugated into Pseudomonas cointegrates. The addition of 3-methylbenzoate for the induction of I-Scel expression was omitted, as the basal expression level was sufficient. Kanamycin-selective clones were directly isolated, positive clones were cured of pSW-2 and restreaked several times. The gene deletion was confirmed by colony PCR and Sanger sequencing. No complementation studies were performed because it has been shown for the I-Scel 
TABLE 5 Primers used in this study

\begin{tabular}{|c|c|c|}
\hline Primer & Sequence $\left(5^{\prime}-3^{\prime}\right)^{a}$ & Description \\
\hline SN019 & gataacagggtaatctgCGCAGGATGAAAGCTAAACC & TS1 ndh-1 forward \\
\hline SN020 & aacagccaTGAGTCGTTCGAATAACTAC & TS1 ndh-1 reverse \\
\hline SN021 & acgactcaTGGCTGTTCAGCAATGATGG & TS2 ndh-1 forward \\
\hline SN022 & cgggtaccgagctcgGCAAGGGCGAGCATGATGAC & TS2 ndh-1 reverse \\
\hline SN023 & gataacagggtaatctgTCGACCTCAACACGCACTTC & TS1 ndh-2 forward \\
\hline SN024 & ccggcaagCGATGCGATGAGTCATGG & TS1 ndh-2 reverse \\
\hline SN025 & tcgcatcgCTTGCCGGCGATAAAGCCAG & TS2 ndh-2 forward \\
\hline SN026 & cgggtaccgagctcgCGGCACTCCCAGATAACTTG & TS2 ndh-2 reverse \\
\hline SN027 & ATACGGGCCGTTCATCAGTC & Verification cointegration ndh-1 forward \\
\hline SN028 & GCGATCTTGCGAATGGTGTC & Verification cointegration ndh-1 reverse \\
\hline SN029 & CCGGCTGAATGACGAATG & Verification cointegration ndh-2 forward \\
\hline SN030 & GTTACGACCCGGTGTATG & Verification cointegration ndh-2 reverse \\
\hline SN112 & tcagataactTTAAGTAGAGCGGCGACTTG & TS1 nuo reverse \\
\hline SN113 & agggataacagggtaatctgCCTTATCGCCGCCGAATCAC & TS1 nuo forward \\
\hline SN114 & ctctacttaaAGTTATCTGAACGGGCTTGG & TS2 nuo forward \\
\hline SN115 & atccccgggtaccgagctcgGCGCTCCAGTTGGTGGATTC & TS2 nuo reverse \\
\hline SN116 & CTCGTCCAAGCCACCTGATG & Verification cointegration nuo forward \\
\hline SN117 & AGCCTCAAGGTCATGGTCTG & Verification cointegration nuo reverse \\
\hline SN171 & CGGACACAGACCATGCATAC & Verification cointegration, binding in nuoA \\
\hline SN200 & CTGCACACCTATGCCTACAA & qPCR ndh-2 forward \\
\hline SN201 & TACAGCGACACATAGAACATCC & qPCR ndh-2 reverse \\
\hline SN214 & TTGGCCCAGAGGAAATCAC & qPCR rpoB forward \\
\hline SN215 & GGCACCGACGTAGACAATAC & qPCR rpoB reverse \\
\hline SN234 & AGAACGAACCCTTCGAATCC & qPCR nuoA forward \\
\hline SN235 & GCATCGCGACCAGATAGAAT & qPCR nuoA reverse \\
\hline SN228 & CGAATACGTCGCTAGCCATAC & qPCR ndh-1 forward \\
\hline SN229 & ATCACTTTCAGGTGCTCGTC & qPCR ndh-1 reverse \\
\hline
\end{tabular}

aUnderlined nucleotides refer to gene-specific regions, and lowercase letters indicate overhangs. The primer efficiencies of the qPCR primer pairs for ndh-2 (SN200, SN201), rpoB (SN214, SN215), nuoA (SN234, SN235), and ndh-1 (SN228 and SN229) were 104.4\%, 101.8\%, 104.4\%, and 103.4\%, respectively.

scarless gene deletion method we used that the double-strand breaks do not result in random mutations. Moreover, the method does not introduce foreign DNA material, making side effects very unlikely.

Analytical methods. The optical density of cell suspensions was measured at a wavelength of $600 \mathrm{~nm}$ using an Ultrospec 10 spectrophotometer (GE Healthcare, Chicago, IL, USA). The cell dry weight $(C D W)$ was calculated by multiplying $\mathrm{OD}_{600 \mathrm{~nm}}$ with a gravimetrically determined correlation factor of 0.39. For HPLC analysis the samples were centrifuged at $17,000 \times g$ for $5 \mathrm{~min}$ and the supernatant was stored at $-20^{\circ} \mathrm{C}$ until further analysis.

Glucose and gluconate concentrations were measured by high-performance liquid chromatography using a Beckman System Gold 126 solvent module equipped with a System Gold 166 UV-detector (Beckman Coulter) and a Smartline RI detector 2300 (KNAUER Wissenschaftliche Geräte, Berlin, Germany). Analytes were separated on the organic resin column Metab AAC (ISERA, Düren, Germany) and eluted with $5 \mathrm{mM} \mathrm{H}_{2} \mathrm{SO}_{4}$ at an isocratic flow of $0.6 \mathrm{ml} \mathrm{min}^{-1}$ at $40^{\circ} \mathrm{C}$ for $20 \mathrm{~min}$. Glucose and gluconate were analyzed using the RI detector, whereas gluconate was determined with the UV detector at a wavelength of $210 \mathrm{~nm}$.

Proteomic profiling of NADH dehydrogenase mutants. Samples for proteome profiling were taken during early, mid-, and late exponential growth at an $\mathrm{OD}_{600 \mathrm{~nm}}$ of $0.5,2.5$, and after depletion of glucose, respectively, and checked with test strips for rapid detection of glucose (Medi-Test; Macherey-Nagel, Düren, Germany). Proteins were extracted from cell biomass and subsequently prepared for shotgun proteomic experiments as described previously (64). All samples were analyzed on an Agilent 6550 iFunnel Q-TOF mass spectrometer (Agilent Technologies, Santa Clara, CA) coupled to an Agilent 1290 UHPLC system. Peptides $(20 \mu \mathrm{g})$ were separated on a Sigma-Aldrich Ascentis Peptides ES-C18 column $\left(2.1 \mathrm{~mm} \times 100 \mathrm{~mm}, 2.7 \mu \mathrm{m}\right.$ particle size, operated at $60^{\circ} \mathrm{C}$ ) at a $0.400 \mathrm{ml} \mathrm{min}^{-1}$ flow rate and eluted with a gradient as follows. The initial condition was $95 \%$ solvent A $(0.1 \%$ formic acid) and $5 \%$ solvent B $(99.9 \%$ acetonitrile, $0.1 \%$ formic acid). Solvent B was increased to $35 \%$ over $120 \mathrm{~min}$, and then increased to $50 \%$ over $5 \mathrm{~min}$, then up to $90 \%$ over $1 \mathrm{~min}$, and held for $7 \mathrm{~min}$ at a flow rate of $0.6 \mathrm{ml} \mathrm{min}-1$, followed by a ramp back down to $5 \% \mathrm{~B}$ over $1 \mathrm{~min}$, where it was held for $6 \mathrm{~min}$ to reequilibrate the column to original conditions. Peptides were introduced to the mass spectrometer from the LC by using a Jet Stream source (Agilent Technologies) operating in positive-ion mode (3,500 V). Source parameters employed gas temperature $\left(250^{\circ} \mathrm{C}\right)$, drying gas $\left(14\right.$ liters $\left.\mathrm{min}^{-1}\right)$, nebulizer $(35 \mathrm{psig})$, sheath gas temperature $\left(250^{\circ} \mathrm{C}\right)$, sheath gas flow (11 liters min $^{-1}$ ), VCap (3,500 V), fragmentor (180 V), and OCT 1 RF Vpp (750 V). The data were acquired with Agilent MassHunter Workstation Software, LC/MS Data Acquisition B.06.01 operating in Auto MS/MS mode, whereby the 20 most intense ions (charge states, 2 to 5) within 300 to 1,400 m/z mass range above a threshold of 1,500 counts were selected for tandem mass spectrometry (MS-MS) analysis. MS-MS spectra (100 to $1,700 \mathrm{~m} / \mathrm{z}$ ) were collected with the quadrupole set to "medium" resolution and were acquired until 45,000 total counts were collected or for a maximum accumulation time of $333 \mathrm{~ms}$. Former parent ions were excluded for $0.1 \mathrm{~min}$ following MS-MS acquisition. The acquired 
data were exported as mgf files and searched against the pan proteome that is highly related to Pseudomonas taiwanensis VLB120 with Mascot search engine version 2.3.02 (Matrix Science). The resulting search results were filtered and analyzed by Scaffold v 4.3.0 (Proteome Software Inc.). The normalized spectral counts of each sample were exported from Scaffold, and the relative quantity changes of identified proteins in mutant samples were calculated in comparison to the wild-type sample. The statistical significance of these changes and the adjusted $P$ values were evaluated by limma $R$ package. (See below for data availability.)

RNA preparation and analysis. Samples for transcription analysis were taken during early, mid- and late exponential growth at an $\mathrm{OD}_{600 \mathrm{~nm}}$ of approximately 0.5, approximately 2.5, and after glucose depletion, respectively. Prior to RNA isolation, the culture sample was diluted with the DNA/RNA protection reagent of the Monarch Total RNA miniprep kit (New England Biolabs, Ipswich, MA, USA), followed by mechanical lysis with ZR BashingBead lysis tube $(0.5 \mathrm{~mm})$ (Zymo Research, Irvine, CA, USA) for $1 \mathrm{~min}$ using the Mini-Beadbeater-16 (Biospec, Bartlesville, OK, USA). After a centrifugation step at $16,000 \times g$ for 2 min the supernatant was transferred into a new tube. An equal volume of RNA lysis buffer of the Monarch Total RNA miniprep kit was added and the RNA isolation was continued as described in the supplier's manual. After the last elution step, an additional in-tube DNase treatment was done using RNase-free DNase I (New England Biolabs, Ipswich, MA, USA). The final RNA yield and purity were evaluated by the absorption ratio $A_{260} / A_{280}$ measured with a Nanodrop (Thermo Scientific, Rockford, IL, USA). The synthesis of CDNA for reverse transcription was carried out with a Protoscript II first strand cDNA synthesis kit (New England Biolabs, Ipswich, MA, USA) using 120 ng total RNA and $60 \mu \mathrm{M}$ random hexamers. The qPCR analyses were conducted with $5 \mu$ l of the reverse transcription reaction mixture with gene-specific primers (Table 5) and the Luna Universal qPCR Master Mix (New England Biolabs, Ipswich, MA, USA) was used. Primers for qPCR were designed with the PrimerQuest Tool of IDT technologies. Gene expression levels for each individual sample were normalized relative to the internal reference gene $r p o B$ and the wild type in the corresponding growth phase calculated by a mathematical method based on the calculated real-time PCR efficiencies (65). The qPCR was performed with the CFX96 Real-Time PCR Detection System (Bio-Rad, Hercules, CA, USA). All qPCRs were performed in triplicates.

Inverted membrane vesicle preparation and NADH oxidation activity. Cultures were harvested at early exponential growth phase at an optical density $\left(O D_{600 n m}\right)$ of approximately 0.5 , as well as in the late exponential growth phase $\left(O D_{600 \mathrm{~nm}}\right.$ of 3 to 4$)$. Inverted membrane vesicles were prepared as described by Borisov (66). Briefly, cells were centrifuged for $8 \mathrm{~min}$ at 5,000 $\times g$ and resuspended in $2 \mathrm{ml}$ spheroplast buffer (200 mM Tris- $\mathrm{HCl}$ pH 8.0, 2 mM EDTA, 30\% sucrose), centrifuged again, and resuspended in $1 \mathrm{ml}$ spheroplast buffer. Spheroplasts were prepared using lysozyme $(0.03 \mathrm{~g})$ and incubated for $30 \mathrm{~min}$ at room temperature. Spheroplasts were centrifuged for $10 \mathrm{~min}$ at 5,000 $\times g$ and resuspended in $2 \mathrm{ml}$ sonication buffer (100 mM HEPES-KOH pH 7.5, $50 \mathrm{mM} \mathrm{K}_{2} \mathrm{SO}_{4}, 10 \mathrm{mM} \mathrm{MgSO}, 2 \mathrm{mM} \mathrm{DTT}, 0.5 \mathrm{mM}$ PMSF). The vesicles were sonicated (Bioruptor, Diadenode, Belgium) in 4 cycles of $30 \mathrm{~s}$ at high intensity with an intermediate pause of $30 \mathrm{~s}$ in ice water. The inverted membrane vesicles were centrifuged twice for $10 \mathrm{~min}$ at $5,000 \times g$ to remove cell debris. The supernatant was centrifuged for $30 \mathrm{~min}$ at $120,000 \times g$ and the resulting pellet was resuspended in the assay buffer $(25 \mathrm{mM}$ HEPES, $25 \mathrm{mM}$ BIS-TRIS propane $\mathrm{pH}$ 7, $10 \mathrm{mM} \mathrm{MgSO}_{4}$ ).

The freshly prepared inverted membrane vesicles were immediately used for the determination of the NADH oxidation activity, as we observed a rapid activity decline when the membrane samples were stored on ice. $150 \mu \mathrm{l} \mathrm{ml}^{-1}$ isolated membrane fractions were added to the assay buffer, and the reaction was initiated by the addition of $125 \mu \mathrm{M} \mathrm{NADH}$. The total volume of the assay was $200 \mu \mathrm{l}$. The NADH oxidation was monitored over $30 \mathrm{~min}$ at $340 \mathrm{~nm}$ in a Synergy MX microplate reader (BioTek, Winooski, VT, USA). For calculating the specific enzyme activity, we used the NADH molar extinction coefficient $\varepsilon_{\mathrm{NADH}}=6.22 \mathrm{mM}^{-1} \mathrm{~cm}^{-1}$, where one unit of activity was the quantity that catalyzed the oxidation of $1 \mu \mathrm{mol}$ of $\mathrm{NADH}$ per min. The protein concentration was measured with the reducing agent compatible Pierce BCA Protein assay kit (Thermo Scientific, Rockford, IL, USA).

Respiration activity monitoring. The cultivations and measurements of the oxygen transfer rate (OTR) and the carbon dioxide transfer rate (CTR) were performed in a modified RAMOS System, developed by the chair of Biochemical Engineering (RWTH Aachen University) (33, 67). The standard RAMOS for shake flasks is commercially available from the Kühner AG (Birsfelden, Switzerland) or HiTec Zang GmbH (Herzogenrath, Germany). All cultivations were performed in 250-ml Ramos flasks with $10 \%$ (vol/vol) filling volume using MSM medium supplemented with $25 \mathrm{mM}$ glucose. The cultures were inoculated from liquid precultures to an approximate $\mathrm{OD}_{600 \mathrm{~nm}}$ of 0.05 . The OTR and CTR were measured thrice per hour. All experiments were performed in biological duplicates.

Redox cofactor quantification. Samples for redox cofactor analysis were collected from early, mid-, and late exponential growth phase at $\mathrm{OD}_{600 \mathrm{~nm}}$ of approximately $0.6,2.2$, and 4.0, respectively. The samples were rapidly transferred into $15-\mathrm{ml}$ Falcon tubes containing $5 \mathrm{ml}$ of quenching solution (acetonitrile:methanol:water, 40:40:20, vol/vol) with ${ }^{13} \mathrm{C}$-labeled cell extracts at $-40^{\circ} \mathrm{C}$. After three freezethaw cycles, the samples were centrifuged at $13,000 \times g$ for $5 \mathrm{~min}$ and concentrated by evaporating the quenching solvent using a vacuum concentrator (SAVANT SpeedVac; Thermo Fisher Scientific, San Diego, CA, USA) for $5 \mathrm{~h}$ followed by lyophilization (LABCONCO; FreeZone, Kansas City, MO, USA). All dried extracts were stored at $-80^{\circ} \mathrm{C}$ until analysis or resuspended in LC-MS grade water for LC-MS analysis. All redox cofactor metabolites were measured on an AB SCIEX Qtrap1 5500 mass spectrometer (AB SCIEX, Framingham, MA, USA) operated in negative ion and selected multiple reaction monitoring (MRM) mode. The column XSELECT HSS XP $(150 \mathrm{~mm} \times 2.1 \mathrm{~mm} \times 2.5 \mu \mathrm{m})$ (Waters, Milford, MA, USA) with ion-pairing technique was used for the chromatography separation as previously described (68). Peak integration 
and metabolite quantification were performed using an isotope-ratio-based approach on Multi-Quant 3.0.2 (AB SCIEX) software as previously described $(69,70)$.

ROS assay. The ROS-sensitive fluorescent dye $2^{\prime}, 7^{\prime}$-dichlorodihydrofluorescein diacetate $\left(\mathrm{H}_{2}\right.$-DCFA, Sigma-Aldrich, St. Louis, MO, USA) was used to monitor the ROS formation in P. taiwanensis VLB120. Cells were harvested at multiple time points during the growth experiment and centrifuged for $5 \mathrm{~min}$ at $8,000 \times g$. The pellet was washed once in the mineral salt medium described above but lacking nitrogen $(\mathrm{MSM}-\mathrm{N})$ and centrifuged again. The cells were resuspended to an optical density of $\mathrm{OD}_{600 \mathrm{~nm}}$ of $\sim 0.4$ in $1 \mathrm{ml}$ MSM-N but supplemented with $25 \mathrm{mM}$ glucose to allow respiratory activity. The ROS-sensitive $\mathrm{H}_{2}$-DCFA dye was added to the cells to a final concentration of $20 \mu \mathrm{M}$. The cells were incubated in the dark for $30 \mathrm{~min}$ at $30^{\circ} \mathrm{C}$ at $250 \mathrm{rpm}$ for aeration. After the incubation, the cells were immediately analyzed in the microplate reader Synergy MX (BioTek, Winooski, VT, USA). The oxidized fluorescent DCF was measured using Ex/Em 504/529 nm. The measurement was performed in biological and technical triplicates. As a positive control, $1 \mathrm{mM} \mathrm{H}_{2} \mathrm{O}_{2}$ was included in all experiments and treated in the same way as the biological samples (data not shown).

Data availability. The mass spectrometry proteomics data have been deposited to the ProteomeXchange Consortium via the PRIDE (71) partner repository with the data set identifier PXD013623.

\section{SUPPLEMENTAL MATERIAL}

Supplemental material is available online only.

SUPPLEMENTAL FILE 1, PDF file, 1.3 MB.

\section{ACKNOWLEDGMENTS}

This study has been conducted within the ERA SynBio project SynPath (grant ID 031A459) with financial support of the German Federal Ministry of Education and Research and is part of the Joint BioEnergy Institute, supported by the Office of Science, Office of Basic Energy Sciences, and Office of Biological and Environmental Research of the U.S. Department of Energy under contract number DE-AC02-05CH11231. B.E.E. and S.C.N. acknowledge financial support by the German Academic Exchange Service (DAAD) through the thematic network Aachen-California Network of Academic Exchange (ACalNet) funded by the German Federal Ministry of Education and Research (BMBF). B.E.E. acknowledges partial support by the CSIRO-UQ Synthetic Biology Alliance. L.M.B. acknowledges funding by the Cluster of Excellence "The Fuel Science Center-Adaptive Conversion Systems for Renewable Energy and Carbon Sources" (EXC 2186), which is funded by the Excellence Initiative of the German federal and state governments to promote science and research at German universities.

We thank Stephani Baum and Uwe Conrath for sharing lab equipment and for technical support. We are grateful to Itay Budin for discussions and instruction on the membrane isolation and in vitro assays and Sophia Nölting for support with qPCR measurements. We thank Benedikt Wynands and Nick Wierckx for sharing the strain P. taiwanensis VLB120 pSTY $^{-}$, Victor de Lorenzo (Centro Nacional de Biotechnología-CNB, Madrid) for providing plasmid pEMG, and Pablo Nikel for providing plasmid pS2311·Nox.

\section{REFERENCES}

1. Kracke F, Kromer JO. 2014. Identifying target processes for microbial electrosynthesis by elementary mode analysis. BMC Bioinformatics 15: 410. https://doi.org/10.1186/s12859-014-0410-2.

2. Kohler KA, Blank LM, Frick O, Schmid A. 2015. D-Xylose assimilation via the Weimberg pathway by solvent-tolerant Pseudomonas taiwanensis VLB120. Environ Microbiol 17:156-170. https://doi.org/10.1111/1462 $-2920.12537$.

3. Becker J, Lange A, Fabarius J, Wittmann C. 2015. Top value platform chemicals: bio-based production of organic acids. Curr Opin Biotechnol 36:168-175. https://doi.org/10.1016/j.copbio.2015.08.022.

4. Yim H, Haselbeck R, Niu W, Pujol-Baxley C, Burgard A, Boldt J, Khandurina J, Trawick JD, Osterhout RE, Stephen R, Estadilla J, Teisan S, Schreyer HB, Andrae S, Yang TH, Lee SY, Burk MJ, Van Dien S. 2011. Metabolic engineering of Escherichia coli for direct production of 1,4-butanediol. Nat Chem Biol 7:445-452. https://doi.org/10.1038/ nchembio.580.

5. Nakamura CE, Whited GM. 2003. Metabolic engineering for the microbial production of 1,3-propanediol. Curr Opin Biotechnol 14:454-459. https://doi.org/10.1016/j.copbio.2003.08.005.

6. Takeno S, Murata R, Kobayashi R, Mitsuhashi S, Ikeda M. 2010. Engineer- ing of Corynebacterium glutamicum with an NADPH-generating glycolytic pathway for L-lysine production. Appl Environ Microbiol 76: 7154-7160. https://doi.org/10.1128/AEM.01464-10.

7. Fasan R, Crook NC, Peters MW, Meinhold P, Buelter T, Landwehr M, Cirino PC, Arnold FH. 2011. Improved product-per-glucose yields in P450dependent propane biotransformations using engineered Escherichia coli. Biotechnol Bioeng 108:500-510. https://doi.org/10.1002/bit.22984.

8. Becker J, Zelder O, Häfner S, Schröder H, Wittmann C. 2011. From zero to hero-design-based systems metabolic engineering of Corynebacterium glutamicum for L-lysine production. Metab Eng 13:159-168. https://doi .org/10.1016/j.ymben.2011.01.003.

9. Berríos-Rivera SJ, Bennett GN, San K-Y. 2002. Metabolic engineering of Escherichia coli: increase of NADH availability by overexpressing an $\mathrm{NAD}^{+}$-dependent formate dehydrogenase. Metab Eng 4:217-229. https://doi.org/10.1006/mben.2002.0227.

10. Jain R, Huang J, Yuan Q, Yan Y. 2015. Engineering microaerobic metabolism of E. coli for 1,2-propanediol production. J Ind Microbiol Biotechnol 42:1049-1055. https://doi.org/10.1007/s10295-015-1622-9.

11. Blank LM, Ebert BE, Bühler B, Schmid A. 2008. Metabolic capacity estimation of Escherichia coli as a platform for redox biocatalysis: constraint- 
based modeling and experimental verification. Biotechnol Bioeng 100 1050-1065. https://doi.org/10.1002/bit.21837.

12. Blank LM, lonidis G, Ebert BE, Bühler B, Schmid A. 2008. Metabolic response of Pseudomonas putida during redox biocatalysis in the presence of a second octanol phase. FEBS J 275:5173-5190. https://doi.org/ 10.1111/j.1742-4658.2008.06648.x.

13. Julsing MK, Kuhn D, Schmid A, Bühler B. 2012. Resting cells of recombinant $E$. coli show high epoxidation yields on energy source and high sensitivity to product inhibition. Biotechnol Bioeng 109:1109-1119. https://doi.org/10.1002/bit.24404.

14. Ebert BE, Kurth F, Grund M, Blank LM, Schmid A. 2011. Response of Pseudomonas putida KT2440 to increased NADH and ATP demand. Appl Environ Microbiol 77:6597-6605. https://doi.org/10.1128/AEM.05588-11.

15. Rühl J, Schmid A, Blank LM. 2009. Selected Pseudomonas putida strains able to grow in the presence of high butanol concentrations. Appl Environ Microbiol 75:4653-4656. https://doi.org/10.1128/AEM.00225-09.

16. Wynands B, Lenzen C, Otto M, Koch F, Blank LM, Wierckx N. 2018. Metabolic engineering of Pseudomonas taiwanensis VLB120 with minimal genomic modifications for high-yield phenol production. Metab Eng 47:121-133. https://doi.org/10.1016/j.ymben.2018.03.011.

17. Wierckx NJP, Ballerstedt H, de Bont JAM, Wery J. 2005. Engineering of solvent-tolerant Pseudomonas putida S12 for bioproduction of phenol from glucose. Appl Environ Microbiol 71:8221-8227. https://doi.org/10 .1128/AEM.71.12.8221-8227.2005.

18. Tiso T, Sabelhaus $P$, Behrens B, Wittgens A, Rosenau F, Hayen H, Blank LM. 2016. Creating metabolic demand as an engineering strategy in Pseudomonas putida-rhamnolipid synthesis as an example. Metab Eng Commun 3:234-244. https://doi.org/10.1016/j.meteno.2016.08.002.

19. Dong J, Chen Y, Benites VT, Baidoo EEK, Petzold CJ, Beller HR, Eudes A, Scheller HV, Adams PD, Mukhopadhyay A, Simmons BA, Singer SW. 2019. Methyl ketone production by Pseudomonas putida is enhanced by plantderived amino acids. Biotechnol Bioeng 116:1909-1922. https://doi.org/ 10.1002/bit.26995.

20. Park JB, Bühler B, Panke S, Witholt B, Schmid A. 2007. Carbon metabolism and product inhibition determine the epoxidation efficiency of solvent-tolerant Pseudomonas sp. strain VLB120 $\Delta$ C. Biotechnol Bioeng 98:1219-1229. https://doi.org/10.1002/bit.21496.

21. Foster JW, Park YK, Penfound T, Fenger T, Spector MP. 1990. Regulation of NAD metabolism in Salmonella typhimurium: molecular sequence analysis of the bifunctional nadR regulator and the nadA-pnuC operon. J Bacteriol 172:4187-4196. https://doi.org/10.1128/JB.172.8.4187-4196 .1990 .

22. Spehr V, Schlitt A, Scheide D, Guénebaut V, Friedrich T. 1999. Overexpression of the Escherichia coli nuo-operon and isolation of the overproduced NADH:ubiquinone oxidoreductase (complex I). Biochemistry 38: 16261-16267. https://doi.org/10.1021/bi9919605.

23. Pruss BM, Nelms JM, Park C, Wolfe AJ. 1994. Mutations in NADH: ubiquinone oxidoreductase of Escherichia coli affect growth on mixed amino acids. J Bacteriol 176:2143-2150. https://doi.org/10.1128/JB.176 8.2143-2150.1994.

24. Matsushita K, Ohnishi T, Kaback HR. 1987. NADH-ubiquinone oxidoreductases of the Escherichia coli aerobic respiratory chain. Biochemistry 26:7732-7737. https://doi.org/10.1021/bi00398a029.

25. Melo AMP, Bandeiras TM, Teixeira M. 2004. New insights into type II $\mathrm{NAD}(\mathrm{P}) \mathrm{H}$ :quinone oxidoreductases. Microbiol Mol Biol Rev 68:603-616. https://doi.org/10.1128/MMBR.68.4.603-616.2004.

26. Spero MA, Aylward FO, Currie CR, Donohue TJ. 2015. Phylogenomic analysis and predicted physiological role of the proton-translocating NADH:quinone oxidoreductase (complex I) across bacteria. mBio 6:e00389-15. https://doi.org/10.1128/mBio.00389-15.

27. Martínez-García E, de Lorenzo V. 2011. Engineering multiple genomic deletions in Gram-negative bacteria: analysis of the multi-resistant antibiotic profile of Pseudomonas putida KT2440. Environ Microbiol 13: 2702-2716. https://doi.org/10.1111/j.1462-2920.2011.02538.x.

28. Torres A, Kasturiarachi N, DuPont M, Cooper VS, Bomberger J, Zemke A. 2019. NADH dehydrogenases in Pseudomonas aeruginosa growth and virulence. Front Microbiol 10:75. https://doi.org/10.3389/fmicb.2019 .00075 .

29. Wynands B, Otto M, Runge N, Preckel S, Polen T, Blank LM, Wierckx N. 2019. Streamlined Pseudomonas taiwanensis VLB120 chassis strains with improved bioprocess features. ACS Synth Biol 8:2036-2050. https://doi .org/10.1021/acssynbio.9b00108.

30. Vicente M, Canovas JL. 1973. Glucolysis in Pseudomonas putida: physi- ological role of alternative routes from the analysis of defective mutants. J Bacteriol 116:908-914. https://doi.org/10.1128/JB.116.2.908-914.1973.

31. del Castillo T, Ramos JL, Rodriguez-Herva JJ, Fuhrer T, Sauer U, Duque E. 2007. Convergent peripheral pathways catalyze initial glucose catabolism in Pseudomonas putida: genomic and flux analysis. J Bacteriol 189:5142-5152. https://doi.org/10.1128/JB.00203-07.

32. Jeude M, Dittrich B, Niederschulte H, Anderlei T, Knocke C, Klee D, Büchs J. 2006. Fed-batch mode in shake flasks by slow-release technique. Biotechnol Bioeng 95:433-445. https://doi.org/10.1002/bit.21012.

33. Anderlei T, Zang W, Papaspyrou M, Büchs J. 2004. Online respiration activity measurement (OTR, CTR, RQ) in shake flasks. Biochem Eng J 17:187-194. https://doi.org/10.1016/S1369-703X(03)00181-5.

34. Calhoun MW, Gennis RB. 1993. Demonstration of separate genetic loci encoding distinct membrane-bound respiratory $\mathrm{NADH}$ dehydrogenases in Escherichia coli. J Bacteriol 175:3013-3019. https://doi.org/10.1128/JB .175.10.3013-3019.1993.

35. Bennett BD, Kimball EH, Gao M, Osterhout R, Van Dien SJ, Rabinowitz JD. 2009. Absolute metabolite concentrations and implied enzyme active site occupancy in Escherichia coli. Nat Chem Biol 5:593-599. https://doi .org/10.1038/nchembio.186.

36. Akkaya Ö, Pérez-Pantoja DR, Calles B, Nikel PI, de Lorenzo V. 2018. The metabolic redox regime of Pseudomonas putida tunes its evolvability toward novel xenobiotic substrates. mBio 9:e01512-18. https://doi.org/ 10.1128/mBio.01512-18.

37. Yu J, Bryant AP, Marra A, Lonetto MA, Ingraham KA, Chalker AF, Holmes DJ, Holden D, Rosenberg M, McDevitt D. 2001. Characterization of the Streptococcus pneumoniae NADH oxidase that is required for infection. Microbiology 147:431-438. https://doi.org/10.1099/00221287 $-147-2-431$.

38. Nikel PI, Pérez-Pantoja D, de Lorenzo V. 2016. Pyridine nucleotide transhydrogenases enable redox balance of Pseudomonas putida during biodegradation of aromatic compounds. Environ Microbiol 18: 3565-3582. https://doi.org/10.1111/1462-2920.13434.

39. Kanehisa M, Sato Y, Kawashima M, Furumichi M, Tanabe M. 2016. KEGG as a reference resource for gene and protein annotation. Nucleic Acids Res 44:D457-D462. https://doi.org/10.1093/nar/gkv1070.

40. Mercenier A, Simon JP, Vander Wauven C, Haas D, Stalon V. 1980. Regulation of enzyme synthesis in the arginine deiminase pathway of Pseudomonas aeruginosa. J Bacteriol 144:159-163. https://doi.org/10 .1128/JB.144.1.159-163.1980.

41. Gonzalez CF, Ackerley DF, Lynch SV, Matin A. 2005. ChrR, a soluble quinone reductase of Pseudomonas putida that defends against $\mathrm{H}_{2} \mathrm{O}_{2}$. J Biol Chem 280:22590-22595. https://doi.org/10.1074/jbc.M501654200.

42. Mailloux RJ, Lemire J, Appanna VD. 2011. Metabolic networks to combat oxidative stress in Pseudomonas fluorescens. Antonie Van Leeuwenhoek 99:433-442. https://doi.org/10.1007/s10482-010-9538-x.

43. Imlay JA. 2003. Pathways of oxidative damage. Annu Rev Microbiol 57:395-418. https://doi.org/10.1146/annurev.micro.57.030502.090938.

44. Messner KR, Imlay JA. 1999. The identification of primary sites of superoxide and hydrogen peroxide formation in the aerobic respiratory chain and sulfite reductase complex of Escherichia coli. J Biol Chem 274: 10119-10128. https://doi.org/10.1074/jbc.274.15.10119.

45. Murphy MP. 2009. How mitochondria produce reactive oxygen species. Biochem J 417:1-13. https://doi.org/10.1042/BJ20081386.

46. Vinogradov AD, Grivennikova VG. 2016. Oxidation of NADH and ROS production by respiratory complex I. Biochim Biophys Acta 1857: 863-871. https://doi.org/10.1016/j.bbabio.2015.11.004.

47. Vilcheze C, Weinrick B, Leung LW, Jacobs WR, Jr. 2018. Plasticity of Mycobacterium tuberculosis NADH dehydrogenases and their role in virulence. Proc Natl Acad Sci U S A 115:1599-1604. https://doi.org/10 .1073/pnas.1721545115.

48. Ahn S, Jung J, Jang IA, Madsen EL, Park W. 2016. Role of glyoxylate shunt in oxidative stress response. J Biol Chem 291:11928-11938. https://doi .org/10.1074/jbc.M115.708149.

49. Kornberg HL. 1966. The role and control of the glyoxylate cycle in Escherichia coli. Biochem J 99:1-11. https://doi.org/10.1042/bj0990001.

50. Lemire J, Alhasawi A, Appanna VP, Tharmalingam S, Appanna VD. 2017. Metabolic defence against oxidative stress: the road less travelled so far. J Appl Microbiol 123:798-809. https://doi.org/10.1111/jam.13509.

51. Kohlstedt M, Wittmann C. 2019. GC-MS-based ${ }^{13} \mathrm{C}$ metabolic flux analysis resolves the parallel and cyclic glucose metabolism of Pseudomonas putida KT2440 and Pseudomonas aeruginosa PAO1. Metab Eng 54:35-53. https://doi.org/10.1016/j.ymben.2019.01.008.

52. Thomas SC, Alhasawi A, Auger C, Omri A, Appanna VD. 2016. The role of 
formate in combatting oxidative stress. Antonie Van Leeuwenhoek 109: 263-271. https://doi.org/10.1007/s10482-015-0629-6.

53. Van Acker H, Sass A, Bazzini S, De Roy K, Udine C, Messiaen T, Riccardi G, Boon N, Nelis HJ, Mahenthiralingam E, Coenye T. 2013. Biofilm-grown Burkholderia cepacia complex cells survive antibiotic treatment by avoiding production of reactive oxygen species. PLoS One 8:e58943. https:// doi.org/10.1371/journal.pone.0058943.

54. Somerville GA, Proctor RA. 2009. At the crossroads of bacterial metabolism and virulence factor synthesis in staphylococci. Microbiol Mol Biol Rev 73:233-248. https://doi.org/10.1128/MMBR.00005-09.

55. Somerville GA, Said-Salim B, Wickman JM, Raffel SJ, Kreiswirth BN, Musser JM. 2003. Correlation of acetate catabolism and growth yield in Staphylococcus aureus: implications for host-pathogen interactions. Infect Immun 71:4724-4732. https://doi.org/10.1128/IAl.71.8.4724-4732 2003.

56. Thauer RK, Jungermann K, Decker K. 1977. Energy conservation in chemotrophic anaerobic bacteria. Bacteriol Rev 41:100-180. https://doi .org/10.1128/MMBR.41.1.100-180.1977.

57. Fernández M, Zúñiga M. 2006. Amino acid catabolic pathways of lactic acid bacteria. Crit Rev Microbiol 32:155-183. https://doi.org/10.1080/ 10408410600880643

58. Segura A, Godoy P, van Dillewijn P, Hurtado A, Arroyo N, Santacruz S, Ramos J-L. 2005. Proteomic analysis reveals the participation of energyand stress-related proteins in the response of Pseudomonas putida DOT-T1E to toluene. J Bacteriol 187:5937-5945. https://doi.org/10.1128/ JB.187.17.5937-5945.2005.

59. Ramos JL, Duque E, Rodríguez-Herva J-J, Godoy P, Haïdour A, Reyes F, Fernández-Barrero A. 1997. Mechanisms for solvent tolerance in bacteria. J Biol Chem 272:3887-3890. https://doi.org/10.1074/jbc.272.7.3887.

60. Sambrook J, Fritsch EF, Maniatis T. 1982. Molecular cloning: a laboratory manual. Cold Spring Harbor Laboratory, Cold Spring Harbor, NY.

61. Hartmans S, Smits JP, van der Werf MJ, Volkering F, de Bont JA. 1989. Metabolism of styrene oxide and 2-phenylethanol in the styrenedegrading Xanthobacter strain 124X. Appl Environ Microbiol 55: 2850-2855. https://doi.org/10.1128/AEM.55.11.2850-2855.1989.

62. Choi KH, Kumar A, Schweizer HP. 2006. A 10-min method for preparation of highly electrocompetent Pseudomonas aeruginosa cells: application for DNA fragment transfer between chromosomes and plasmid transformation. J Microbiol Methods 64:391-397. https://doi.org/10.1016/j .mimet.2005.06.001.

63. Chomczynski P, Rymaszewski M. 2006. Alkaline polyethylene glycolbased method for direct PCR from bacteria, eukaryotic tissue samples, and whole blood. Biotechniques 40:454-458. https://doi.org/10.2144/ 000112149.

64. Gonzalez Fernandez-Nino SM, Smith-Moritz AM, Chan LJ, Adams PD,
Heazlewood JL, Petzold CJ. 2015. Standard flow liquid chromatography for shotgun proteomics in bioenergy research. Front Bioeng Biotechnol 3:44. https://doi.org/10.3389/fbioe.2015.00044.

65. Pfaffl MW. 2001. A new mathematical model for relative quantification in real-time RT-PCR. Nucleic Acids Res 29:e45. https://doi.org/10.1093/nar/ 29.9.e45.

66. Borisov VB, Murali R, Verkhovskaya ML, Bloch D, Han H, Gennis RB, Verkhovsky MI. 2011. Aerobic respiratory chain of Escherichia coli is not allowed to work in fully uncoupled mode. Proc Natl Acad Sci U S A 108:17320-17324. https://doi.org/10.1073/pnas.1108217108.

67. Schulte A, Schilling JV, Nolten J, Korona A, Krömke H, Vennekötter J-B, Schillheim B, Wessling M, Conrath U, Büchs J. 2018. Parallel online determination of ethylene release rate by Shaken Parsley cell cultures using a modified RAMOS device. BMC Plant Biol 18:101. https://doi.org/ 10.1186/s12870-018-1305-6.

68. McCloskey D, Utrilla J, Naviaux RK, Palsson BO, Feist AM. 2015. Fast Swinnex filtration (FSF): a fast and robust sampling and extraction method suitable for metabolomics analysis of cultures grown in complex media. Metabolomics 11:198-209. https://doi.org/10.1007/s11306 -014-0686-2.

69. Wu L, Mashego MR, van Dam JC, Proell AM, Vinke JL, Ras C, van Winden WA, van Gulik WM, Heijnen JJ. 2005. Quantitative analysis of the microbial metabolome by isotope dilution mass spectrometry using uniformly ${ }^{13} \mathrm{C}$-labeled cell extracts as internal standards. Anal Biochem 336: 164-171. https://doi.org/10.1016/j.ab.2004.09.001.

70. Mashego MR, Wu L, Van Dam JC, Ras C, Vinke JL, Van Winden WA, Van Gulik WM, Heijnen JJ. 2004. MIRACLE: mass isotopomer ratio analysis of $\mathrm{U}-{ }^{13} \mathrm{C}$-labeled extracts. A new method for accurate quantification of changes in concentrations of intracellular metabolites. Biotechnol Bioeng 85:620-628. https://doi.org/10.1002/bit.10907.

71. Perez-Riverol $Y$, Csordas A, Bai J, Bernal-Llinares M, Hewapathirana $S$, Kundu DJ, Inuganti A, Griss J, Mayer G, Eisenacher M, Pérez E, Uszkoreit J, Pfeuffer J, Sachsenberg T, Yılmaz Ş, Tiwary S, Cox J, Audain E, Walzer M, Jarnuczak AF, Ternent T, Brazma A, Vizcaíno JA. 2019. The PRIDE database and related tools and resources in 2019: improving support for quantification data. Nucleic Acids Res 47:D442-D450. https://doi.org/10 $.1093 / \mathrm{nar} / \mathrm{gky} 1106$.

72. Cunin R, Glansdorff N, Piérard A, Stalon V. 1986. Biosynthesis and metabolism of arginine in bacteria. Microbiol Rev 50:314-352. https:// doi.org/10.1128/MMBR.50.3.314-352.1986.

73. Ditta G, Stanfield S, Corbin D, Helinski DR. 1980. Broad host range DNA cloning system for Gram-negative bacteria: construction of a gene bank of Rhizobium meliloti. Proc Nat Acad Sci U S A 77:7347-7351. https://doi .org/10.1073/pnas.77.12.7347. 